\title{
Control of Hox transcription factor concentration and cell-to-cell variability by an auto-regulatory switch
}

\author{
Dimitrios K. Papadopoulos ${ }^{1, * \neq}$, Kassiani Skouloudaki ${ }^{1}$, Ylva Engström², Lars Terenius ${ }^{3}$, Rudolf Rigler ${ }^{4,5}$, \\ Christoph Zechner ${ }^{1,6}$, Vladana Vukojevićc ${ }^{3}$ and Pavel Tomancak ${ }^{1, \ddagger}$
}

\begin{abstract}
The variability in transcription factor concentration among cells is an important developmental determinant, yet how variability is controlled remains poorly understood. Studies of variability have focused predominantly on monitoring mRNA production noise. Little information exists about transcription factor protein variability, as this requires the use of quantitative methods with single-molecule sensitivity. Using Fluorescence Correlation Spectroscopy (FCS), we have characterized the concentration and variability of 14 endogenously tagged TFs in live Drosophila imaginal discs. For the Hox TF Antennapedia, we investigated whether protein variability results from random stochastic events or is developmentally regulated. We found that Antennapedia transitioned from low concentration/high variability early, to high concentration/low variability later, in development. FCS and temporally resolved genetic studies uncovered that Antennapedia itself is necessary and sufficient to drive a developmental regulatory switch from autoactivation to auto-repression, thereby reducing variability. This switch is controlled by progressive changes in relative concentrations of preferentially activating and repressing Antennapedia isoforms, which bind chromatin with different affinities. Mathematical modeling demonstrated that the experimentally supported auto-regulatory circuit can explain the increase of Antennapedia concentration and suppression of variability over time.
\end{abstract}

\section{KEY WORDS: Auto-regulation, Fluorescence correlation spectroscopy, Hox genes, Protein noise, Transcription factors, Variability}

\section{INTRODUCTION}

In order to understand the mechanisms that control pattern formation and cell fate specification in developing organisms, the intranuclear concentration, DNA-binding kinetics and cell-to-cell

\footnotetext{
${ }^{1}$ Max-Planck Institute of Molecular Cell Biology and Genetics, 01307 Dresden, Germany. ${ }^{2}$ Department of Molecular Biosciences, The Wenner-Gren Institute, Stockholm University, 10691 Stockholm, Sweden. ${ }^{3}$ Center for Molecular Medicine (CMM), Department of Clinical Neuroscience, Karolinska Institutet, 17176 Stockholm, Sweden. ${ }^{4}$ Department of Medical Biochemistry and Biophysics, Karolinska Institutet, 17177 Stockholm, Sweden. ${ }^{5}$ Laboratory of Biomedical Optics, Swiss Federal Institute of Technology, 1015 Lausanne, Switzerland. ${ }^{6}$ Center for Systems Biology Dresden, 01307 Dresden, Germany.

*Present address: MRC Human Genetics Unit, Institute of Genetics and Molecular Medicine, University of Edinburgh, Edinburgh EH4 2XU, UK.
}

†Authors for correspondence (dpapado2@ed.ac.uk; tomancak@mpi-cbg.de)

(D) D.K.P., 0000-0003-0914-3051; P.T., 0000-0002-2222-9370

This is an Open Access article distributed under the terms of the Creative Commons Attribution License (https://creativecommons.org/licenses/by/4.0), which permits unrestricted use, distribution and reproduction in any medium provided that the original work is properly attributed.

Received 22 May 2018; Accepted 20 November 2018 variability of relevant transcription factors (TFs) need to be quantified. TF concentration variability at the tissue level is thought to arise from diverse processes, including mRNA transcription, translation and protein degradation. Intrinsic noise is due to stochastic binding and interactions of proteins involved in transcriptional activation of a specific gene (Blake et al., 2003; Elowitz et al., 2002). Extrinsic noise arises from inter-cellular differences in abundance of the transcriptional and posttranscriptional machinery (Swain et al., 2002).

In undifferentiated tissue or cells, TF cell-to-cell variability can be the driving force for differentiation. For example, progressive establishment of a Nanog salt-and-pepper expression pattern leads to the formation of primitive endoderm in the mouse preimplantation embryo, whereas loss of the variability results in embryos lacking primitive endoderm entirely (Kang et al., 2013).

Conversely, in already differentiated tissue or cells, TF expression variability among cells may need to be counteracted to ensure homogeneity of gene expression patterns and robustness of commitment to a certain transcriptional regime. Examples are the Snail (Sna) TF, which is required for the invagination of the mesoderm during Drosophila gastrulation (Boettiger and Levine, 2013), or the Bicoid (Bcd) and Hunchback ( $\mathrm{Hb})$ TFs during early embryogenesis (Gregor et al., 2007a,b; Little et al., 2013).

In addition, differential cell fates within the same developmental territory may be specified by TFs deploying different DNA-binding dynamics, despite the existence of very similar concentrations (i.e. low variability). For example, studies on the Oct4 TF in early mouse embryos have shown that differential kinetic behavior of DNA binding, despite equal Oct4 concentration among blastomeres, ultimately dictates an early developmental bias towards lineage segregation (Kaur et al., 2013; Plachta et al., 2011).

So far, studies of gene expression variability have focused predominantly on monitoring the noise of mRNA production (Holloway et al., 2011; Holloway and Spirov, 2015; Little et al., 2013; Lucas et al., 2013; Paré et al., 2009). Little information exists about TF variability at the protein level within a tissue. Such studies require the use of quantitative methods with single-molecule sensitivity.

We have previously used Fluorescence Correlation Spectroscopy (FCS) to quantitatively characterize Hox TF interactions with chromatin in living salivary gland cells (Papadopoulos et al., 2015; Vukojevic et al., 2010). FCS is instrumental for quantifying TF dynamics in living cells or tissue (Clark et al., 2016; Kaur et al., 2013; Lam et al., 2012; Mistri et al., 2015; Papadopoulos et al., 2015; PerezCamps et al., 2016; Szaloki et al., 2015; Tiwari et al., 2013; Tsutsumi et al., 2016). However, in these studies, only mobility has been measured for overexpressed proteins. To understand TF behavior in vivo, proteins need to be quantified at endogenous levels (Lo et al., 2015).

In this study, we take advantage of the availability of fly toolkits, in which TFs have been endogenously tagged using different 
methodologies, fosmid (Baumgartner et al., 1996), BAC (deposition of lines of Rebecca Spokony and Kevin White to FlyBase and the Bloomington Stock Center), FlyTrap (Buszczak et al., 2007; Kelso et al., 2004; Morin et al., 2001; Quinones-Coello et al., 2007) and MiMIC lines (Nagarkar-Jaiswal et al., 2015; Venken et al., 2011), to measure the intranuclear concentration of various TFs in vivo by FCS, and their cell-to-cell variability in fly imaginal discs. Imaginal discs are flat, single-layered epithelia comprising small diploid cells and many TFs are expressed in defined regions within these tissues during development.

\section{RESULTS}

\section{Characterization of average protein concentrations and} cell-to-cell variability of Drosophila TFs

Average concentrations of TFs in neighboring nuclei of third instar imaginal discs were measured by FCS (Fig. 1A-J and Fig. S1A-P). FCS is a non-invasive method with single-molecule sensitivity, in which a confocal arrangement of optical elements is used to generate a small (sub-femtoliter) detection volume inside living cells, from which fluorescence is being detected (Fig. 1C,D; green ellipsoid). Fluorescent molecules diffuse through this observation volume, yielding fluorescence intensity fluctuations that are recorded over time by detectors with single-photon sensitivity (Fig. 1E). These fluctuations are subsequently subjected to temporal autocorrelation analysis, yielding temporal autocorrelation curves (henceforth referred to as FCS curves, Fig. 1F), which are then fitted with selected models to extract quantitative information about the dynamic processes underlying the generation of the recorded fluctuations. In the case of molecular movement of TFs (see supplementary Materials and Methods), information can be obtained regarding: (1) the absolute TF concentrations (Fig. 1F); (2) TF dynamic properties, such as diffusion times, differences in their interactions with chromatin and fractions of free-diffusing versus chromatin-bound TFs (Fig. 1G); and (3) cell-to-cell TF concentration variability (Fig. 1H).

We selected 14 TFs based on the availability of homozygous endogenously tagged transgenes and on the generation of robust fluorescence in distinct patterns in various imaginal discs. For the $14 \mathrm{TFs}$, we measured average concentrations ranging across about two orders of magnitude among the different TFs, from $\sim 30 \mathrm{nM}$ to $\sim 1.1 \mu \mathrm{M}$ ( $\sim 400$ to 15,500 molecules per nucleus, respectively) (Fig. 1I, Fig. S1A-Q and supplementary Materials and Methods). Various diffusion times and fractions of slow- and fast-diffusing TF molecules (Fig. 1J) indicated differential mobility and degree of DNA-binding among different TFs (Vukojevic et al., 2010). Comparison of the $y$-axis amplitudes at the zero lag time of the FCS curves, which are inversely proportional to the concentration of fluorescent molecules (Fig. 1F), provides information about concentration variability (heterogeneity) among different cell nuclei, i.e. reflects the heterogeneity of protein concentration at the tissue level (Fig. 1H). For all 14 TFs studied, the variability, expressed as the variance over the mean squared, $C V^{2}=\frac{s^{2}}{m^{2}}$, was determined to be in the range $7-37 \%$ (Fig. 1K and Fig. S1Q).

In biological systems, the Fano factor, which is expressed as the variance over the mean $\left(F_{f}=\frac{s^{2}}{m}\right.$, in concentration units), is a commonly used index to quantify variability. It has been proposed that Fano factor values that increase with average concentrations indicate that the underlying transcriptional processes cannot be sufficiently explained by a simple one-step promoter configuration with purely intrinsic Poissonian noise and that extrinsic noise is likely to contribute significantly to the overall variability (Newman et al., 2006; Schwanhäusser et al., 2011; Taniguchi et al., 2010). For all TFs measured, Fano factor values from 0 to 20 were obtained (Fig. S1R), in line with Fano factor values of other TFs determined previously to lie between 0 and 30 (Sanchez et al., 2011). Moreover, the majority of TFs examined show Fano factor values, $F_{\mathrm{f}}>1$, suggesting that transcriptional bursting is likely to be a significant source of the observed cell-to-cell variability. We used this dataset as a starting point for studying the control of variability during imaginal disc development.

The average concentration and variability of the investigated TFs showed no obvious interdependence (Fig. 1K), suggesting that if variability is controlled, there is not one control mechanism that is common to all investigated TFs. Among the studied TFs, the Hox protein Antennapedia (Antp) showed low variability $\left(C V^{2}<0.2\right)$ in high average concentrations, in particular in the leg disc (Fig. 1K). As low variability at the tissue level is likely to be achieved through regulatory mechanisms, we investigated Antp variability further by FCS. Because FCS performs best at low to moderate expression levels (see supplementary Materials and Methods), we performed this analysis in the wing disc where expression levels are lower than in the leg disc (Fig. 1K,L). We first established that the observed fluorescence intensity fluctuations were caused by diffusion of TF molecules through the confocal detection volume (Figs S2 and S1). FCS showed that different clusters of neighboring cells along the Antp expression domain in the wing disc display different average expression levels (Fig. 1L). Moreover, FCS showed that Antp cell-to-cell variability decreased with increasing Antp concentration (Fig. 1M), whereas the Fano factor increased (Fig. S1R). Such behavior is indicative of complex transcriptional regulatory processes (Franz et al., 2011; Smolander et al., 2011) that we further investigated using the powerful Drosophila genetic toolkit.

\section{Control of Antp concentration by transcriptional auto-regulation}

One mechanism by which genes control their expression level variability is auto-regulation (Becskei and Serrano, 2000; Dublanche et al., 2006; Gronlund et al., 2013; Nevozhay et al., 2009; Shimoga et al., 2013; Thattai and van Oudenaarden, 2001). To test whether Antp can regulate its own protein levels, we monitored the concentration of endogenous Antp protein upon overexpression of Antp from a transgene. To distinguish between overexpressed and endogenous protein, we used synthetic Antp (SynthAntp) transgenes fused to eGFP (SynthAntp-eGFP). These transgenes encode the Antp protein (amino acids 278-378), which includes the homeodomain, the conserved YPWM motif and the $\mathrm{C}$ terminus (but lack the long and non-conserved $\mathrm{N}$ terminus of the protein, against which widely used Antp antibodies have been raised) and they harbor Antp-specific homeotic function (Papadopoulos et al., 2011). Clonal overexpression of SynthAntp$e G F P$ in the wing disc notum (Fig. $2 \mathrm{~A}, \mathrm{~B}^{\prime}, \mathrm{D}$ and controls in Fig. S3D, $\mathrm{D}^{\prime}$ ) repressed the endogenous Antp protein, indicating that Antp is indeed able to regulate its own protein levels.

As Antp is a TF, we next asked whether the auto-repression occurs at the transcriptional level. The Antp locus is subject to complex transcriptional regulation, involving a distal and a proximal promoter ( $\mathrm{P} 1$ and $\mathrm{P} 2$ promoters, respectively), spanning more than $100 \mathrm{~kb}$ of regulatory sequences. We established that the $\mathrm{P} 1$ promoter (rather than the $\mathrm{P} 2$ promoter) is predominantly required to drive expression of Antp in the wing disc notum (Fig. S3A-C'), in line with previous observations (Engstrom et al., 1992; Jorgensen and Garber, 1987; Zink et al., 1991) (see Materials and Methods). Moreover, mitotic recombination experiments in regions of the wing disc unique to $\mathrm{P} 2$ transcription have shown no function of the $\mathrm{P} 2$ 
A

Imaginal disc

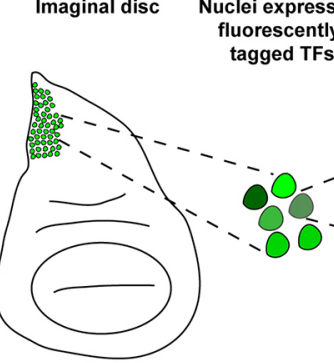

$\mathbf{F}$

B

Concentration
FCS measurement in a small volume in the nucleus

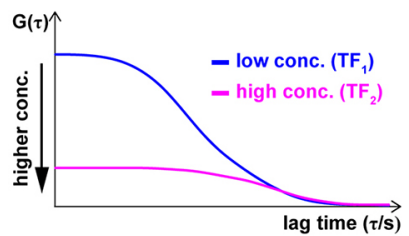

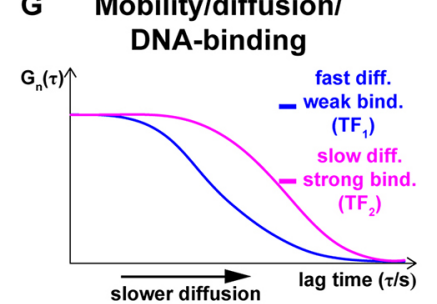

slower diffusion
D

Confocal detection volum (sub-femtoliter)

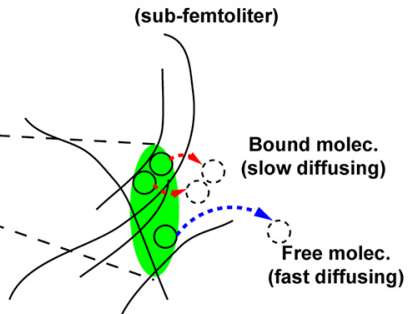

\section{E}

Fluorescence intensity fluctuations

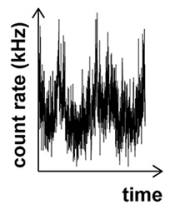

H Variability

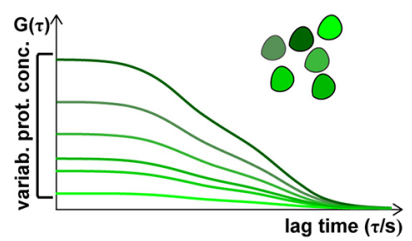

All TFs

I

$J$

K

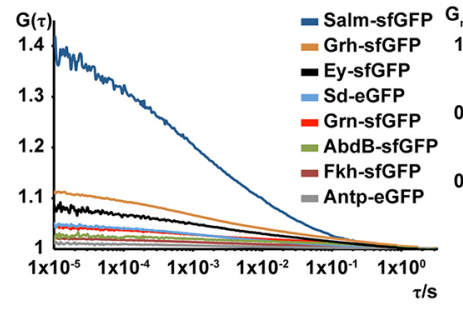

$G_{n}(\tau)$
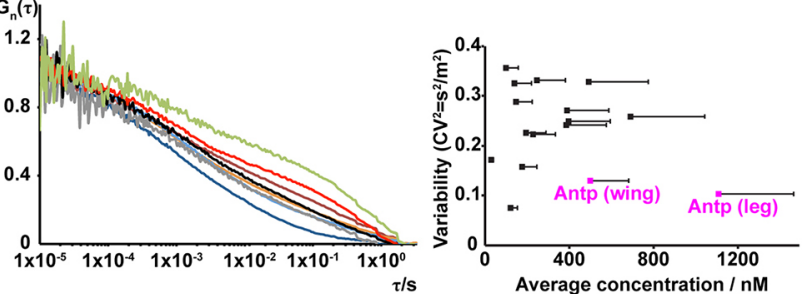

Antennapedia (Antp)

L

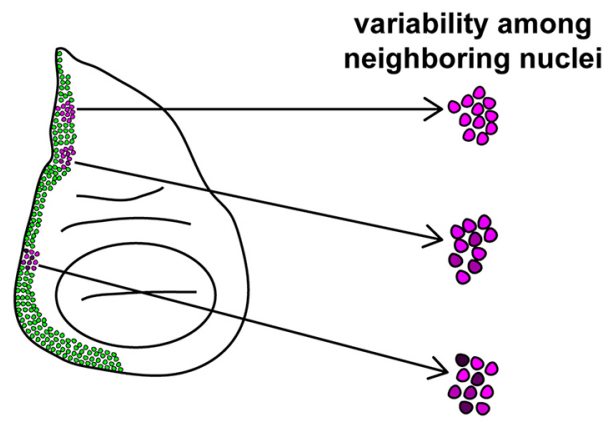

M

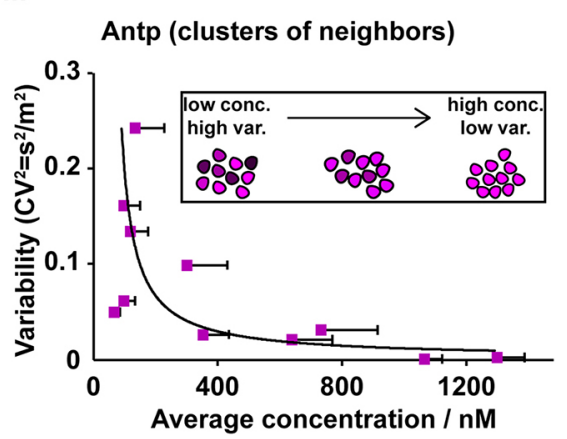

Fig. 1. Concentration, DNA-binding dynamics and cell-to-cell protein concentration variability of 14 Drosophila TFs. (A-H) Workflow of the study of TFs by FCS (see Materials and Methods and supplementary Materials and Methods). (A) Schematic of an imaginal disc with cells expressing an endogenouslytagged TF (green), as imaged by confocal laser scanning microscopy. (B) Schematic of cell nuclei in neighboring cells expressing the TF at different concentrations. (C) Schematic of a cell nucleus with the observation volume element (OVE) for FCS measurements in the form of a prolate ellipsoid depicted as a green ellipse. (D) Magnified drawing of the OVE shown in $\mathrm{C}$ and its immediate surrounding. (E) Fluorescence intensity fluctuations occurring at fast and slow timescales are generated by TF molecules quickly/slowly diffusing into/out of the OVE. (F) After deploying temporal autocorrelation analysis to examine the fluorescence intensity fluctuations, temporal autocorrelation curves (henceforth referred to as FCS curves) are generated, which, after fitting with an appropriate model function, yield information about the absolute concentration and diffusion of TFs, as well as the fraction of fast- and slow-diffusing TF molecules. The concentration of molecules is inversely proportional to the $y$-axis amplitude of the FCS curve at zero lag time. (G) FCS curves normalized to the same amplitude. Processes that slow down the diffusion of TF molecules, such as binding to very large molecules (e.g. chromatin), are visible by a shift of the FCS curves to longer lag times. (H) FCS curves recorded in neighboring cell nuclei allow the calculation of protein concentration variability at the live tissue level. Here, the TF concentration is the highest in the brightest green nucleus, corresponding to the FCS curve with the lowest amplitude, and the TF concentration is the lowest in the dark green nucleus, corresponding to the FCS curve with the highest amplitude. (I) Representative average FCS measurements of eight TFs. (J) FCS curves shown in I, normalized to the same amplitude, $G_{n}(\tau)=1$ at $\tau=10 \mu \mathrm{s}$. (K) Variability of the 14 TFs as a function of concentration. (L) Variability in concentration of endogenous Antp in the wing disc. Antp protein distribution in third instar wing imaginal disc (green). Examples of different regions in the disc, analyzed by FCS (magenta), displaying different variabilities in the concentration of nuclear Antp (darker and lighter magenta shades). (M) Variability of Antp concentration in clusters of neighboring cell nuclei as a function of its average concentrations. Error bars in $\mathrm{K}$ and $\mathrm{M}$ represent $1 \mathrm{~s} . \mathrm{d}$.

promoter transcripts in wing disc development (Abbott and Kaufman, 1986). Thus, the P1 Antp reporter serves as a suitable reporter of the Antp locus transcriptional activity in this context.
Clonal overexpression of SynthAntp-eGFP in the wing disc repressed the Antp P1 transcriptional reporter (Fig. 2C,D and controls in Fig. S3E, $\mathrm{E}^{\prime}$ ). To rule out putative dominant-negative 


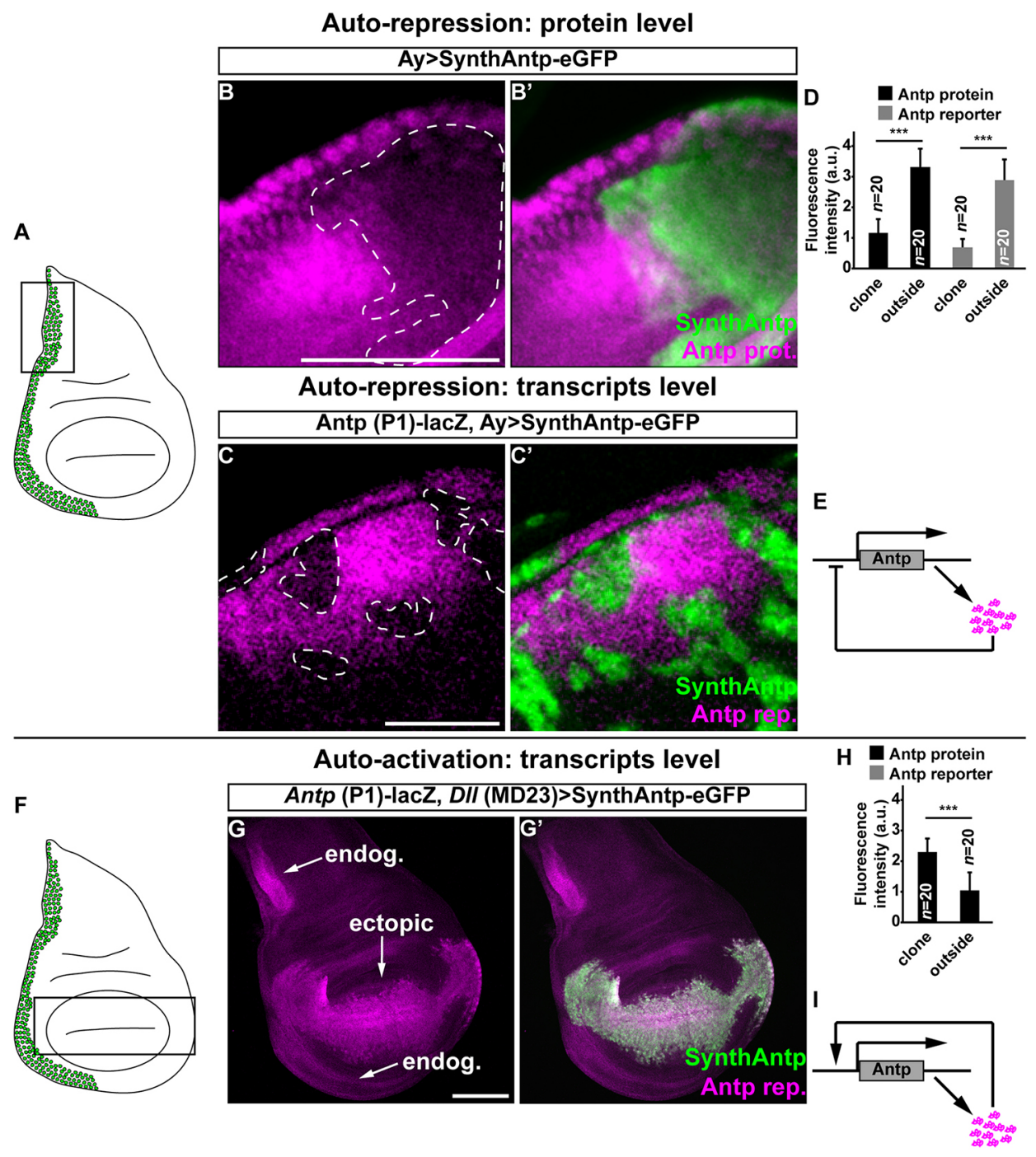

Fig. 2. Antp activates and represses its own transcription. (A) Schematic representation of the wing disc region of highest Antp expression (green cells). Antp is highly expressed in the wing disc in the regions of the notum that correspond to the structure of the prescutum in the adult cuticle, as well as in the base of the wing blade, which gives rise to the mesopleura and pteropleura of the adult thoracic cuticle. The black rectangle indicates the region of clonal analysis in $B-C^{\prime}$.

$\left(\mathrm{B}, \mathrm{B}^{\prime}\right)$ Clonal overexpression of a SynthAntpeGFP construct. Dashed line in B shows a clone in the Antp expression domain.

$\left(C, C^{\prime}\right)$ Transcriptional auto-repression of Antp using the Antp P1-lacZ. (D) Quantification of repression of Antp protein and reporter inside the repression clones, when compared with the surrounding tissue. (E) Schematic of Antp transcriptional auto-repression. Repression can be direct or indirect. $(F)$ Wing disc region of ectopic Antp P1 reporter expression in G, G $\left(\mathrm{G}, \mathrm{G}^{\prime}\right)$ Ectopic induction of Antp P1-lacZ in distal compartments of the wing disc by expression of SynthAntp-eGFP using DII-Gal4 (MD23). (H) Quantification of auto-activation of Antp reporter within the DII-Gal4 expression domain when compared with the surrounding tissue. (I) Schematic representation of Antp auto-activation. Scale bars: $100 \mu \mathrm{m}$.

activity of the small SynthAntp-eGFP peptide, we also performed these experiments with the full-length Antp protein (Fig. S3F,F') and found them to also repress the reporter. We conclude that the Antp protein is able to repress its own transcription from the P1 promoter (directly or indirectly), suggesting a possible mechanism of suppressing cell-to-cell variability of Antp expression levels (Fig. 2E).

In the course of these experiments, we noticed that ectopic overexpression of SynthAntp-eGFP or the full-length Antp protein from the Distal-less (Dll) (MD23) enhancer resulted in activation of the Antp $\mathrm{P} 1$ reporter in distal compartments of the wing disc, such as the wing pouch, where Antp is normally not detected (Fig. 2F-H and controls in Fig. S3G-H'). This suggests that as well as its autorepressing function, Antp is also capable of activating its own transcription (Fig. 2I).

To exclude the possibility that the auto-activation and repression of Antp are artifacts of overexpression, we used FCS to measure the concentration of Antp triggered by different Gal4 drivers (Fig. S4A-E). We observed indistinguishable DNA-binding behavior by FCS, not only across the whole concentration range examined (Fig. S4F), but also between endogenous and overexpressed Antp (Fig. S5A,B). Importantly, the auto-activating and auto-repressing capacity of Antp was preserved even with the weak Gal4-driver 69B (Fig. S4K,L) that triggered concentrations of Antp lower than its normal concentration in the leg disc (473 nM versus $1110 \mathrm{nM})$, indicating that auto-activation and auto-repression of Antp take place at endogenous protein concentrations. We conclude that Antp is able to repress and activate its own transcription (Fig. 2E,I), and hypothesize that this auto-regulatory circuit sets the 'correct' concentration of Antp protein in imaginal discs.

\section{A temporal switch controls the transition of Antp from a state of auto-activation to a state of auto-repression}

To further investigate the mechanism by which the Antp autoregulatory circuit sets the precise Antp expression levels, we next asked whether the seemingly opposing auto-regulatory activities of Antp are separated in time during development. To achieve this, we induced gain-of-function clones of full-length untagged Antp either at $26 \mathrm{~h}$ (first larval instar - henceforth referred to as 'early' stage) or at $60 \mathrm{~h}$ (late second larval instar - henceforth referred to as 'late' stage) of development and analyzed the clones in late third instar wing imaginal discs (Fig. 3). We chose these time points based on Antp expression being widespread during first instar disc development and therefore possibly amenable to auto-activation before becoming confined to the proximal disc regions, whereas in the late second instar it is restricted to proximal-only regions (Emerald and Cohen, 2004). As a pre-requisite for this analysis, we established that the Antp-eGFP homozygous viable MiMIC 


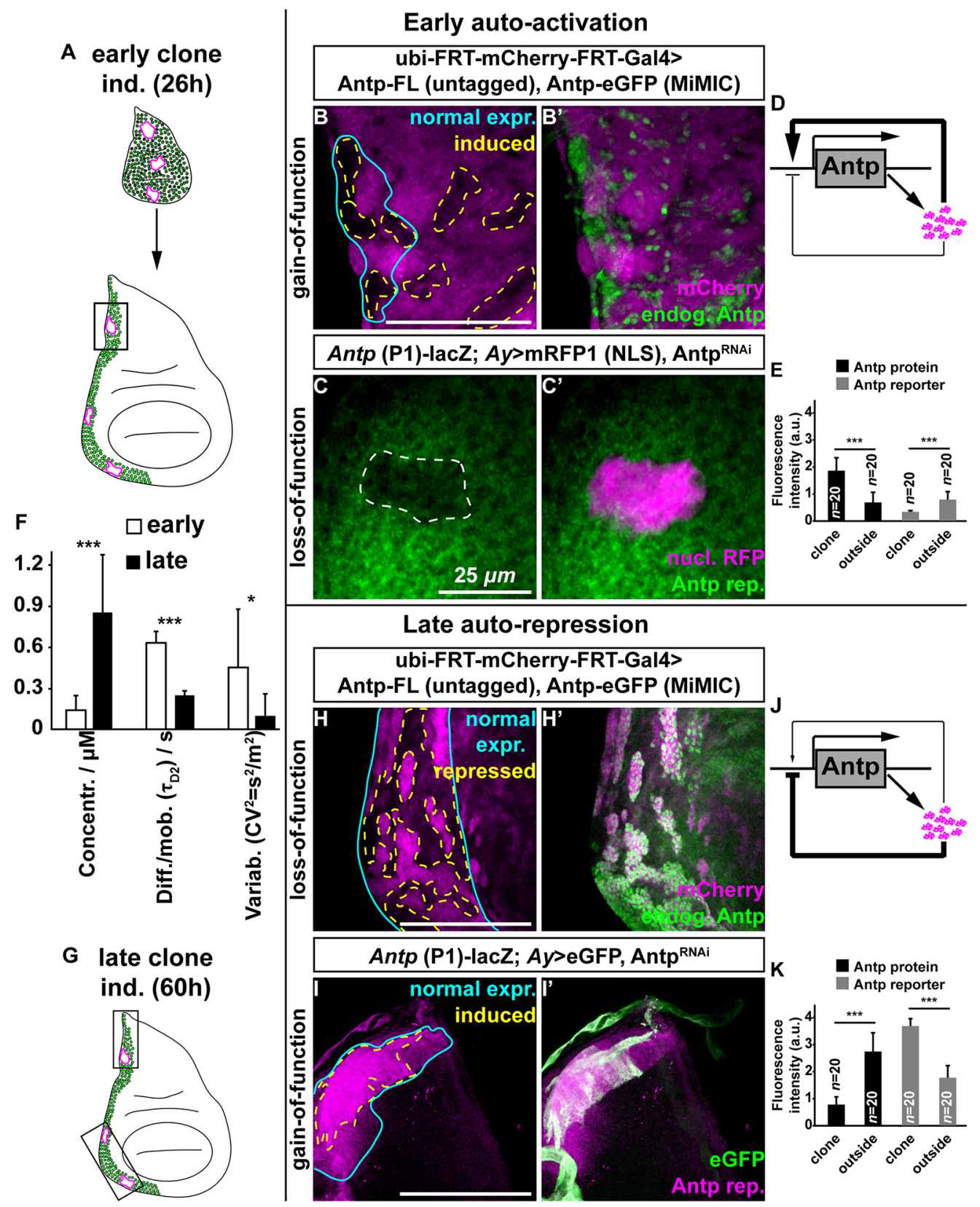

Fig. 3. Antp switches from transcriptional auto-activation to auto-repression. (A) Clone induction at $26 \mathrm{~h}$ (early) with analysis at third instar larval stage ( 96$120 \mathrm{~h}$ of development). Black rectangle represents the corresponding region of clonal analysis. (B, $\mathrm{B}^{\prime}$ ) Early clonal induction of full-length, untagged Antp, (mCherry positive) reveals strong auto-activation of endogenous Antp-eGFP (dashed lines in B). The cyan line outlines the region of highest endogenous Antp expression. The whole Antp expression domain expresses Antp-eGFP, but overexpression clones (subregions marked by absence of mCherry staining) express Antp-eGFP much more strongly ( $\left.\mathrm{B}^{\prime}\right)$. (C, $\left.\mathrm{C}^{\prime}\right)$ Antp P1 transcription in Antp RNAi knockdown clones (early clonal induction, dashed line in C) marked by nuclear mRFP1.

(D) Updated Antp auto-activation model, showing strong auto-activation of Antp at early stages. (E) Quantification of fluorescence intensities (in a.u.) upon early clonal induction of endogenous Antp protein, upregulated upon overexpression of untagged full-length Antp (as in B,B'), and of Antp reporter downregulated upon knockdown by RNAi (as in C,C'). (F) Concentration, DNA-binding and variability studied by FCS at second instar leg and wing discs (FCS analysis in Fig. S8). Low concentration, low degree of DNA-binding and high variability are observed in second instar wing and leg discs, but the opposite is true for third instar discs.

(G) Clone induction at $60 \mathrm{~h}$ (late) with analysis at third instar larval stage ( 96-120 h of development). Black rectangles represent the corresponding regions of clonal analysis. $\left(\mathrm{H}, \mathrm{H}^{\prime}\right)$ Late-induced clones (dashed lines in $\mathrm{H}$ ), expressing full-length, untagged Antp (mCherry positive). Auto-repression of Antp in these regions is monitored by the endogenous Antp-eGFP protein. The cyan lines in $\mathrm{H}$ and I outline the region of strong endogenous expression of the Antp protein ( $\mathrm{H}$ ) and the Antp $\mathrm{P} 1$ reporter (I). (I,I') Antp P1 transcription in late Antp RNAi knockdown clones (dashed line in I, $60 \mathrm{~h}$ of development) within the Antp normal expression domain, marked by nuclear mRFP1. Cytoplasmic eGFP marks the Antp knockdown clone $\left(\mathrm{I}^{\prime}\right)$. (J) Updated Antp auto-repression model showing the pronounced auto-repressing capacity of Antp at late stages. (K) Quantification of fluorescence intensities (in a.u.) upon late clonal induction of endogenous Antp protein, downregulated upon overexpression of untagged full-length $A$ tp (as in $\mathrm{H}, \mathrm{H}^{\prime}$ ), and of Antp reporter upregulated upon knockdown by RNAi (as in I,I'). Scale bars: 100 um.

allele recapitulates the endogenous Antp pattern in the embryo and all thoracic imaginal discs, and therefore can be used to monitor endogenous Antp protein (Fig. S6). Clonal induction of full-length untagged Antp in early development triggered strong auto-activation of Antp-eGFP (Fig. 3A,B, $\mathrm{B}^{\prime}$ and quantification in $\mathrm{E}$, see controls in Fig. S7A-C'). As before, we confirmed that early 
auto-activation of Antp is transcriptional and similar for both fulllength and SynthAntp proteins (Fig. S7D-E', see controls in F-G'). Early auto-activation was further supported by results from a lossof-function experiment, where RNAi-mediated early knockdown of Antp resulted in downregulation of the Antp reporter (Fig. 3C, $\mathrm{C}^{\prime}$, see controls in Fig. $\mathrm{S} 7 \mathrm{H}, \mathrm{H}^{\prime}$ ). The loss- and gain-of-function analysis together suggest that during early disc development Antp is required for sustaining its own expression.

In contrast, clonal induction during the late second instar stage (Fig. 3G) repressed Antp-eGFP (Fig. 3H, $\mathrm{H}^{\prime}$ and quantification in $\mathrm{K}$ ) and, reciprocally, the clonal knockdown by RNAi triggered autoactivation of Antp transcription (Fig. 3I, $\mathrm{I}^{\prime}$ ). Hence, in contrast to early development, Antp represses its own expression in third instar discs. Although the gain-of-function experiments show that Antp is sufficient to execute auto-regulation, loss-of-function analysis indicates that it is also necessary for both repression and activation at the transcriptional level.

Together, these results revealed the existence of a switch in Antp auto-regulatory capacity on its own transcription during development. Starting from a preferentially auto-activating state early in development (Fig. 3D), Antp changes to an auto-inhibitory mode at later developmental stages (Fig. 3J).

\section{During development, Antp switches from a Iow-concentration/high-variability to a high-concentration/ low-variability state}

If the Antp auto-repressive state limits the variability of Antp protein concentration among neighboring cells late in development, we expected that the variability would be higher during earlier stages, when auto-repression does not operate. We therefore used FCS to characterize the endogenous expression levels and cell-to-cell variability of Antp concentration in nuclei of second instar wing and leg discs. We observed significantly lower average concentrations of Antp protein in second versus third instar wing and leg discs, and the inverse was true for concentration variability (Fig. $3 \mathrm{E}$ and Fig. S8A, $\mathrm{A}^{\prime}, \mathrm{C}$ ), indicating that the developmental increase in concentration is accompanied by suppression of concentration variability. In addition, FCS revealed a notable change in Antp characteristic decay times (signifying molecular diffusion, limited by chromatin binding) at early versus late stages (Fig. S8B). This behavior indicates that endogenous Antp is initially moving fast in the nucleus, as it undergoes considerably fewer interactions with chromatin compared with later stages where its interactions with chromatin are more frequent and longer lasting.

Taken together, our measurements show that Antp is expressed at relatively low and highly variable levels in early developing discs, when genetic evidence indicates auto-activation capacity on its own transcription. Later in development, when Antp has reached a state of higher average concentrations, auto-repression kicks in, resulting in considerably lower variability among neighboring cells.

\section{Dynamic control of Antp auto-regulation by different Antp isoforms}

The changing binding behavior of Antp on chromatin from second to third instar discs and the developmental transition from an autoactivating to an auto-repressing state suggested a causal relationship between the two phenomena. We therefore sought to identify molecular mechanisms that could link the observed changes in Antp chromatin binding to Antp auto-activation and repression. It is well established that the Antp mRNA contains an alternative splice site in exon 7 immediately upstream of the homeobox-containing exon 8, and generates Antp isoforms differing in as little as four amino acids in the linker between the YPWM motif (a co-factor-interacting motif) and the homeodomain (Fig. 4A) (Stroeher et al., 1988). Our previous observation that long linker isoforms favor transcriptional activation of Antp target genes, whereas short linker isoforms favor repression of Antp targets (Papadopoulos et al., 2011), prompted us to examine whether the linker length is also responsible for differences in auto-regulation.

Ectopic expression of SynthAntp-eGFP peptides featuring a long linker displayed significantly weaker repression capacity on endogenous Antp, when compared with their short linker counterparts (Fig. 4B, $\mathrm{B}^{\prime}, \mathrm{F}, \mathrm{F}^{\prime}$ and quantified in $\mathrm{D}, \mathrm{H}$, see also Materials and Methods). We confirmed that, also in this case, the repression was at the transcriptional level (Fig. S9I-J'). Inversely, long-linker Antp isoforms exhibited stronger activation of Antp reporter, when compared with short-linker isoforms (Fig. 4C, $\mathrm{C}^{\prime}, \mathrm{G}$, $\mathrm{G}^{\prime}$ and quantified in $\mathrm{D}, \mathrm{H}$; see also Materials and Methods). We additionally validated that short-linker isoforms encoded by fulllength or SynthAntp cDNAs behaved as weaker auto-activating and stronger auto-repressing Antp species in all our previous experiments using the endogenous Antp protein and the P1 reporter (Fig. S9A-H'). We conclude that, also in the case of Antp auto-regulation, short-linker isoforms function as more potent repressors, whereas long-linker isoforms operate as more potent activators.

As the Antp P1 promoter undergoes a switch from preferential auto-activation to auto-repression, and short- and long-linker Antp isoforms function as preferential auto-repressors and autoactivators, respectively, it appeared possible that the switch in Antp regulation is executed at the level of transcript variant abundance of these isoforms. Therefore, we next quantified the relative abundance of long- and short-linker transcript variants in the embryo, second and third instar discs (Fig. 4D,H). The data showed that the abundance of the long-linker variant decreased, whereas the abundance of the short-linker variant increased over time in development, in line with previous observations (Stroeher et al., 1988). Thus, as hypothesized, this finding suggests that relative transcript variant abundance may underlie the switch between auto-activation and auto-repression (without excluding additional mechanisms, such as changes in the chromatin modifications between early and later disc development, or the participation of different co-factors).

Relative changes in Antp transcript variant abundance (Fig. 4D,H), differential efficiency of their encoded isoforms to repress or activate the Antp gene (Fig. 4B-D,F-H), the developmental switch of Antp from auto-activation to repression (Fig. 3) and the different mobility of Antp between second and third instar imaginal discs (Fig. 3E) all pointed towards the hypothesis that the two isoforms have different modes of interaction with chromatin. To investigate this, we expressed the two isoforms from the $69 \mathrm{~B}$ enhancer in third instar wing and antennal discs. This results in Antp concentrations close to (if not below) endogenous levels (Fig. S4A-J). FCS measurements revealed that the short-linker isoform displayed longer characteristic decay times and a higher fraction of DNA-bound molecules, suggesting stronger and more pronounced binding to chromatin than its long-linker counterpart (Fig. 4D,H and Fig. S10A,B). With chromatin (and therefore Antp-binding sites configuration), as well as the presence of co-factor proteins, being identical between the two instances (short- and long-linker isoforms examined in third instar wing and antennal imaginal discs of the same age), we were able to directly compare the apparent equilibrium dissociation constants for the two isoforms (see supplementary Materials and Methods). We found that the affinity 
A
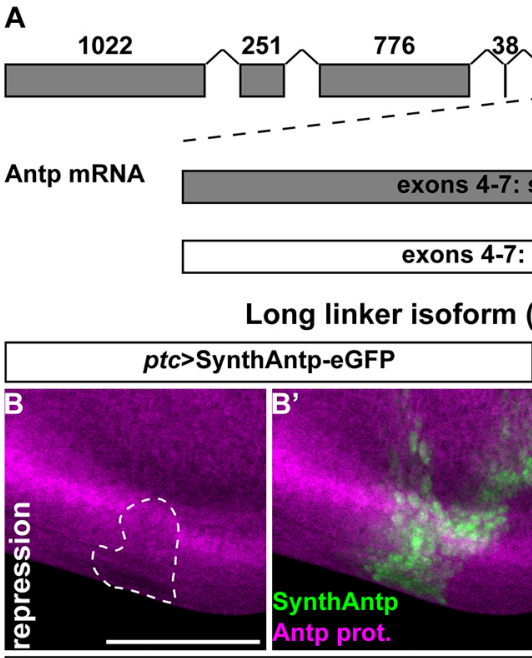

225/ homeobox

Antp (P1)-lacZ; DII (MD23)>SynthAntp -eGFP
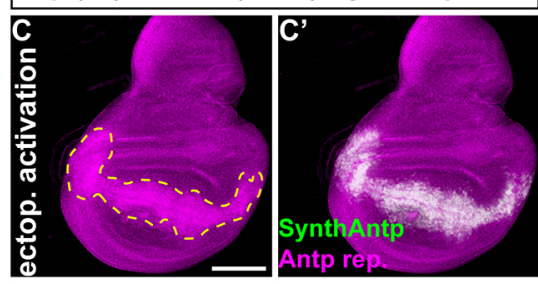

Short linker isoform (preferential repressor)
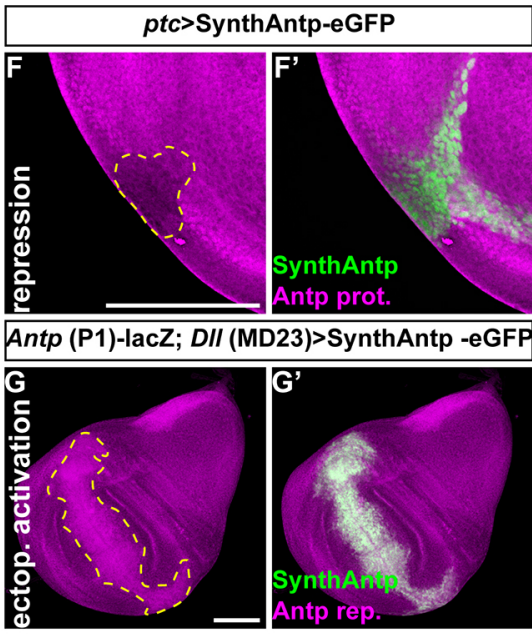

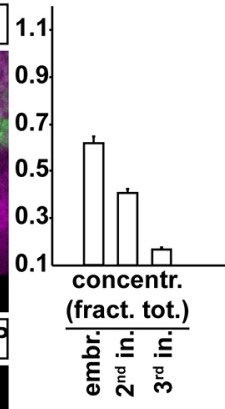

$\mathrm{E}$

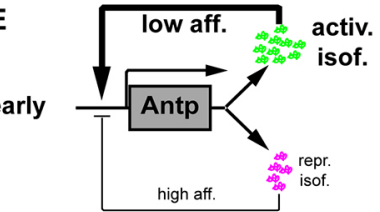

2285

YPWM-RSQF

Iternative splice sites in the linker

$\mathrm{H}$

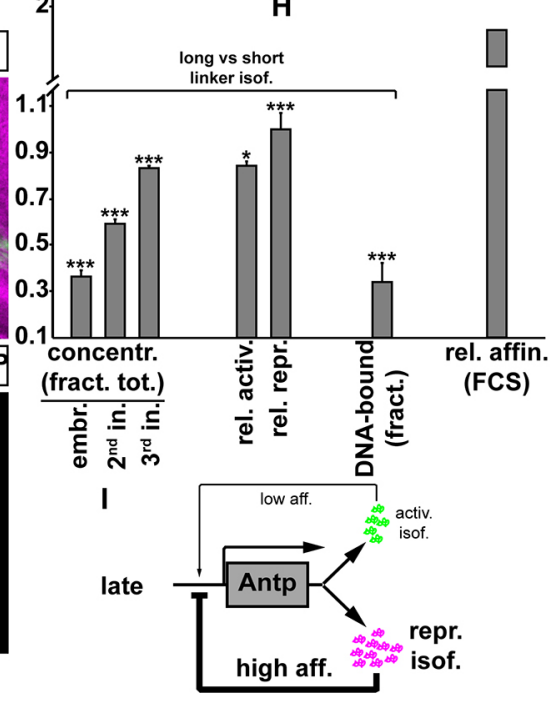

Fig. 4. Antp auto-activation and auto-repression relies on Antp isoforms with different binding affinities to chromatin. (A) Schematic of the Antp mRNA, generated from the $\mathrm{P} 1$ promoter. Exons are represented by gray boxes Magnified exons 4-7 (drawn to scale, omitting splicing points for simplicity) show the alternative splice site ( $3^{\prime}$ of exon 7 ), resulting in isoforms featuring a short linker between the YPWM motif and the homeodomain (RSQF, gray box) or a long linker isoform (RSQFGKCQ, white box).

(B, $\left.\mathrm{B}^{\prime}\right)$ SynthAntp-eGFP bearing a long linker expressed by ptc-Gal4 and endogenous Antp protein auto-repression were monitored at the proximal region of the wing disc. A white dashed line outlines the region of auto-repression that was used for quantification (see Materials and Methods).

$\left(C, C^{\prime}\right)$ Similar to $B, B^{\prime}$, except that expression was induced by DII (MD23)-Gal4 distally (yellow dashed line).

(D) Abundance of long linker isoform (see Materials and Methods); auto-activation and auto-repression efficiencies (Materials and Methods); DNA-bound fractions, measured by FCS (Fig. S10); and relative affinity of binding to chromatin, calculated by FCS (Fig. S10) are presented for comparison with H. (E) Updated model of Antp autoregulation. The activating isoform binds with lower affinity to the P1 Antp promoter, but is produced in excess, relative to the repressing isoform, resulting in preferential activation of transcription. (F-G') Similar to B-C' for the short linker isoform. (H) Similar to D for comparison. (I) Updated qualitative model representation of Antp repression as in $\mathrm{E}$, whereby at later stages an excess of Antp auto-repressor accounts for negative feedback on transcriptional regulation of the P1 promoter, resulting in partial activation of transcription; hence, expression is maintained. Scale bars: $100 \mu \mathrm{m}$ of binding to chromatin $\left(K_{d}^{-1}\right)$ of the repressing short-linker isoform was at least 2.3 times higher compared with the activating long-linker isoform $\quad\left(\frac{K_{d, \text { Antp long linker issorm }}}{K_{d, \text { Ant short linker issorm }}}>2.3\right.$ ) (Fig. $4 \mathrm{D}, \mathrm{H}$ and Fig. S10C-D ${ }^{\prime}$ ). To corroborate these findings, we also performed gel-shift experiments to test how full-length recombinant Antp isoforms, which bear a short and a long linker, bind previously characterized Antp-binding sites. We found that equal amounts of Antp long-linker isoform bind Antp-binding sites more weakly than its short linker counterpart (Fig. S11). Collectively, these experiments support the notion that differences in Antp regulation during disc development can be largely attributed to differences in the affinity of the investigated Antp isoforms.

Taken together, the switch of Antp from an auto-activating to an auto-repressing state and the alteration of its DNA-binding behavior during disc development can be largely explained by a temporal developmental regulation of the relative concentrations of preferentially auto-activating and auto-repressing Antp protein isoforms. These isoforms display distinct properties in their modes of interaction with chromatin (Fig. 4E,I).

\section{Robustness of Antp auto-regulation}

The mechanism of developmental Antp auto-regulation offered a possible explanation for the observed increase in Antp concentration from second to third instar discs, as well as the suppression of variability. An unresolved issue is the functional significance of suppression of Antp variability in development. To test this, we need to manipulate variability, yet this is currently not possible to achieve at the endogenous locus. However, as average concentration and variability are interdependent, we used an ectopic expression system to progressively dampen Antp variability by 
manipulating its concentration. To achieve this, we expressed SynthAntp ectopically in the antennal disc, which is devoid of endogenous Antp expression, and monitored the extent (strength) of homeotic transformations induced by different Gal4 drivers corresponding to different SynthAntp concentrations (as measured by FCS previously in Fig. S4A-D). In this experiment, expression of SynthAntp is controlled by the Gal4 driver, independently of the Antp locus, therefore the phenotypic output does not depend on Antp auto-regulation. We observed that partial transformations of antennae to tarsi could be obtained with drivers expressing Antp at close to endogenous concentration [ptc-Gal4, Dll-Gal4 (MD713) and $69 \mathrm{~B}-\mathrm{Gal} 4$ drivers, Fig. 5B-D and Fig. S4B-D]. Therefore, Antp can repress the antennal and launch the leg developmental program in the antennal disc at endogenous concentrations, although not robustly across the tissue (see supplementary Materials and Methods and Table S1 for analysis of the phenotypic classes). As expected, the three weak transformation phenotypes, elicited by ptc-, Dll (MD713)- and 69B-Gal4 (Fig. 5B-D), were accompanied by high variability of SynthAntp concentration in developing discs (Fig. 5E,F). In contrast, strong expression of SynthAntp from the Dll-Gal4 (MD23) enhancer resulted in robust homeotic transformation to a complete tarsus (Fig. 5A), accompanied by low cell-to-cell variability (Fig. 5F). This condition resembled most closely the endogenous Antp variability in the leg disc
$\left(C V^{2}=0.103\right)$. Importantly, endogenous Antp and Antp overexpressed by any of the Gal4 drivers showed indistinguishable chromatin-binding behavior by FCS (Figs S4F and S5A,B). Therefore, robust Antp homeotic function can be achieved at concentrations that are accompanied by low variability.

In order to further substantiate the qualitative model of Antp auto-regulation suggested by our findings and examine its impact on protein variability, we developed a simple mathematical model of stochastic Antp expression (see supplementary Materials and Methods and Table S2). This model tests whether positive and negative auto-regulation of Antp through distinct isoforms is sufficient to explain the increase in protein concentration and decrease in nucleus-to-nucleus variability from early to late stages. The model consists of a dynamic promoter, which drives transcription of Antp followed by a splicing step, yielding either the auto-repressing or the auto-activating isoform of Antp. As the repressing isoform has higher abundance at later stages, we assumed that splicing is more likely to generate this isoform than the activating isoform. The initial imbalance of Antp towards the activating isoform (Fig. 4D,H) is modeled through appropriate initial concentrations of each isoform.

As Antp copy numbers per nucleus are in the thousands at both early and late stages of development, the intrinsic noise of gene expression is likely to explain only a specific part of the overall
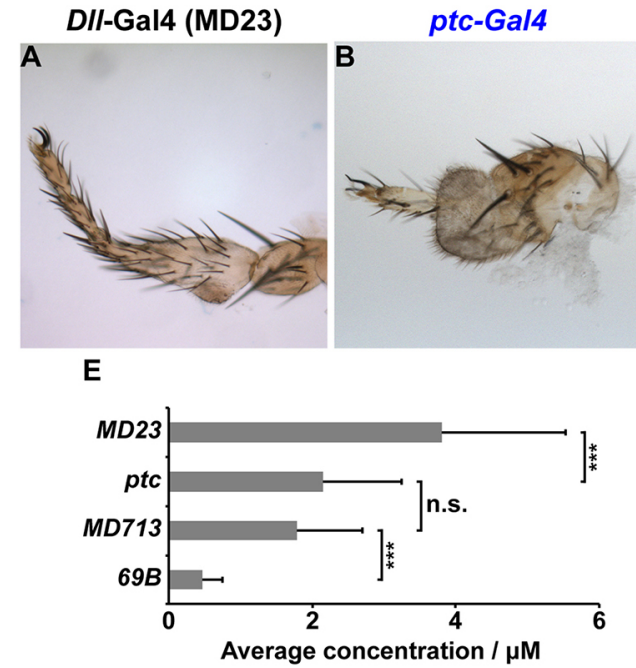

$\mathbf{F}$

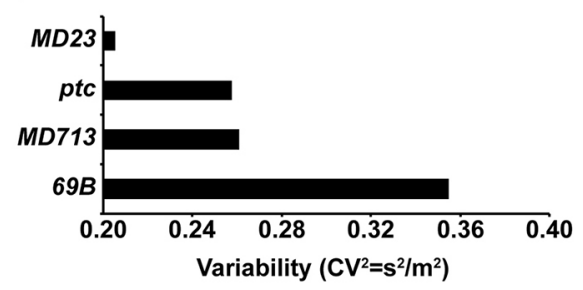

activating isoform repressing isoform

G

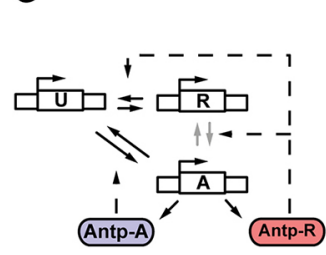

H
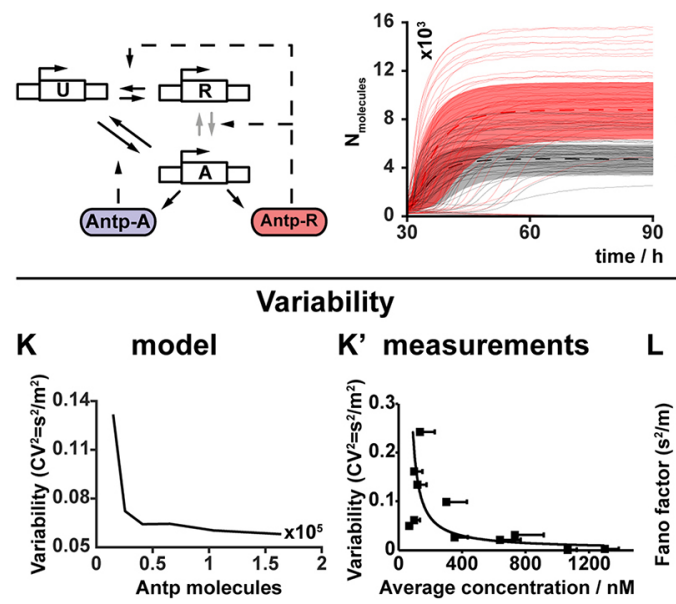

K' measurements L

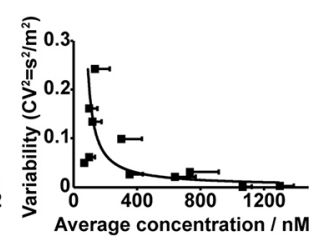

I
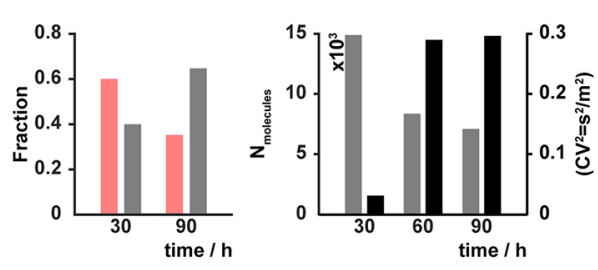

DII-Gal4 (MD713)

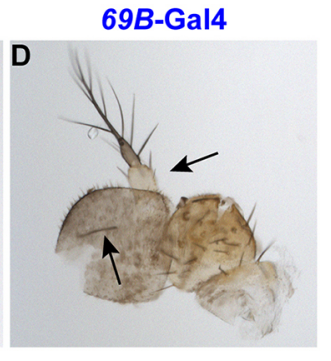

Fano factor
L' measurements
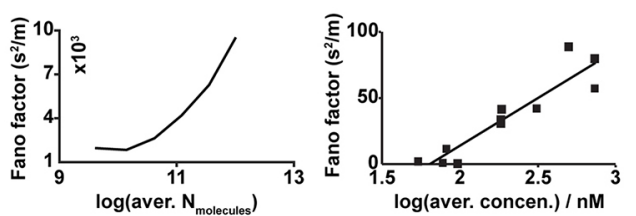

Fig. 5. Concentrations resulting in low variability are required for Antp homeotic function. (A-D) Transformations of the distal antenna into a tarsus in adult flies, caused by SynthAntp-eGFP overexpression in antennal discs (Fig. S4A-D). Ectopic tarsi range from complete $(A)$ to milder transformations of the arista $(B, C)$ or ectopic leg bristles in the third antennal segment (C,D, arrows). (E,F) Measurements of SynthAntp concentration and cell-to-cell variability of antennal discs (Fig. S4A-D) in the corresponding antennal discs (A-D). The three Gal4 drivers (B-D) result in partial transformations, despite being expressed at similar levels to the wild-type Antp protein in the leg disc. However, their variability is higher than the endogenous variability $\left(C V^{2}=0.1\right)$. In contrast, Dll-Gal4 (MD23) results in much more robust homeotic transformations $(A)$, accompanied by the lowest variability and closest to the wild-type condition. (G-J) A dynamic promoter, which drives transcription of Antp followed by a splicing step, leads to either the repressing (' $R$ ' in $G$ ) or activating ('A' in $G$ ) isoform of Antp. In the absence of Antp, the promoter is inactive and transcription cannot take place ('U' in G). This promoter configuration leads to suppression of variability and increase in Antp concentration (J). Trajectories of individual simulations are presented in $\mathrm{H}$ and the distribution of the Antp isoforms, predicted by the model, are presented in I. (K-L') Model predictions $(\mathrm{K}, \mathrm{L})$ and experimental data validation $\left(K^{\prime}, L^{\prime}\right)$ of variability $(\mathrm{K})$ and protein Fano factor $(\mathrm{L})$ as a function of Antp concentration. 
variability in Antp concentrations (Elowitz et al., 2002; Taniguchi et al., 2010). The remaining extrinsic variability is due to cell-to-cell differences in certain factors affecting gene expression such as the ribosomal or ATP abundances. To check whether extrinsic variability significantly affects Antp expression, we expressed nuclear $m R F P 1$ constitutively, alongside endogenous Antp-eGFP, and measured their abundances (Fig. S12). With extrinsic factors affecting both genes similarly, we expected a correlation between the concentration of nuclear mRFP1 and Antp-eGFP. Our data showed a statistically significant correlation between mRFP1 and Antp (Fig. S11C, $r=0.524$ and $P=9.7710^{-5}$ ). Correspondingly, we accounted for extrinsic variability also in our model by allowing gene expression rates to randomly vary between cells (Zechner et al., 2012).

The promoter itself is modeled as a Markov chain with three distinct transcriptional states. In the absence of Antp, the promoter is inactive and transcription cannot take place (state ' $U$ ' in Fig. 5G). It can switch into a highly expressing state ' $\mathrm{A}$ ' at a rate that is assumed to be proportional to the concentration of the auto-activating isoform (Antp-A, Fig. 5G). This resembles the positive autoregulatory function of Antp. Conversely, the promoter can be repressed by recruitment of the auto-repressing isoform, state ' $R$ ' in the model (Antp-R, Fig. 5G). As the auto-repressing isoform of Antp can also activate the promoter, albeit significantly weaker than the auto-activating isoform, and vice versa, we allow the promoter to switch between states 'A' and ' $R$ '.

In this promoter model, it remains unclear whether the two isoforms compete for the same binding sites on the P1 promoter. In this case, an increase in concentration of repressing Antp species enhances the probability to reach state ' $R$ ' only if the promoter is in state 'U' (Fig. 5G). In the absence of competitive binding, the rate of switching between ' $A$ ' and ' $R$ ' also depends on the concentration of repressing isoforms of Antp (Fig. 5G, compare with Fig. S13A). We analyzed both model variants by forward simulation and found that both of them can explain the switch-like increase in average Antp concentration between early and late stages (Fig. 5J, compare with Fig. S13D) and the relative fraction of repressing and activating isoforms (Fig. 5I, compare with Fig. S13C). However, only the non-competitive binding model (Fig. 5G) can explain the substantial reduction of total Antp variability between early and late stages (Fig. 5J, Fig. S13D). Simulation trajectories of individual nuclei indicated an initial increase and a subsequent stabilization of concentration, whereas in the competitive model, or in the absence of the negative feedback, this is not achieved (Fig. 5H, compare with Fig. S13B,F). Additionally, we established that the negative feedback is required for suppression of variability (Fig. S13E,H), as otherwise no suppression of variability is conferred (Fig. S13H). Thus, the model suggested that auto-repression is required and that isoforms do not compete for binding to the $\mathrm{P} 1$ promoter.

To further validate this model, we analyzed how Antp variability scales with average concentrations, compared with our experimental measurements. To generate different average concentrations, we varied the gene expression rates over three orders of magnitude. The model predicted a decrease in variability as a function of total Antp concentration and an increase in the Fano factor. These findings are in good agreement with the experimental data (compare Fig. $5 \mathrm{~K}$ with $\mathrm{K}^{\prime}$ and $\mathrm{L}$ with $\mathrm{L}^{\prime}$ ).

We next analyzed the model behavior under different genetic perturbations. Increase of Antp concentration by overexpressing SynthAntp transgenes (bearing either a long or a short linker isoform) from the Antp P1 promoter (Antp P1-Gal4>SynthAntp$e G F P$ long or short linker) resulted in $100 \%$ embryonic lethality, rendering the analysis of concentration and variability in imaginal discs impossible. This indicated that an indiscriminate increase of the dose of either Antp variant from early embryonic development onwards cannot be tolerated or buffered by the auto-regulatory circuit.

However, overexpression from a Dll enhancer [Dll-Gal4 (MD23)] in the leg discs or in the notum (MS243-Gal4), which overlaps with the endogenous Antp expression pattern only during first instar disc development (Emerald and Cohen, 2004), resulted in normal adult leg and notum structures. Flies overexpressing either the SynthAntp auto-activating or the auto-repressing isoform in distal appendages (Fig. 6A,B) or the notum (Fig. S14A) displayed the wild-type morphology, indicative of normal Antp function, regardless of which isoform (activating or repressing) was overexpressed. We further measured by FCS the concentration and variability of the total Antp protein (endogenous Antp-eGFP and overexpressed SynthAntp-eGFP) in proximal regions of the leg disc at second and third instar stages (Fig. 6C, $\mathrm{C}^{\prime}$ ). We found that the concentration remained high at both stages due to overexpression, but variability was reduced to endogenous levels at late stages. In addition, the reduced Antp variability does not seem to depend on Antp concentration alone, because for high concentrations at both early and late stages, variability is high only in the early stage and reduced in the late stage. Together, the phenotypic analysis and FCS measurements indicate that Antp auto-regulation is able to reduce variability, even at high levels of expression of either isoform, ensuring proper leg development.

The experimental data were corroborated by the model, which predicted that more than threefold overexpression of either autoactivating or auto-repressing Antp isoforms (Fig. 6E,H) will nevertheless equilibrate to normal expression levels at later stages (Fig. 6D,F,G,I). Specifically, we have measured by FCS roughly 15,400 molecules in the wild-type leg disc, and the model is in good quantitative agreement with this measurement upon overexpression of the activating or repressing isoform. In addition, there is no negative effect on the noise-suppressing property of the circuit (Fig. 6F,I). Thus, both the model and experimental data indicate that transient high levels of either isoform early during disc development can be tolerated and that the concentration and cell-to-cell variability of the endogenous Antp protein is restored at later stages.

In contrast, overexpression of an exogenous repressor, such as Sex combs reduced (Scr), which can repress Antp at the transcriptional level, but can neither activate it nor activate its own transcription (Fig. S14E-J'), resulted in abnormal leg (Fig. 6J) and notum (Fig. S14B) development. These flies died as pharate adults with malformed legs, in line with Antp being required for proper leg development in all ventral thoracic discs (legs). FCS measurements in the corresponding proximal leg disc cell nuclei of second and third instar leg discs overexpressing mCherry-SynthScr revealed pronounced reduction in Antp concentration and remarkable increase in variability (Fig. 6K). In agreement, the model predicted a similar block of transcription and correspondingly severe effects on Antp dynamics (Fig. 6L-N). In both the measurements and the model prediction, the high increase in variability was triggered by the fact that a majority of the cells do not 'manage' to switch into the highly-expressing state, as too few long-linker Antp molecules are present to establish the positive auto-regulation. Because splicing favors the short-linker isoforms at later stages, these cells never 'recover' from Scr repression after restriction of the Antp overexpression domain to proximal regions of the leg disc (Fig. 6L). Taken together, the minimal model of Antp auto-regulatory genetic circuit is able to explain the experimentally 
Perturbations (constit. activation/repression by Antp isoforms) SynthAntp-eGFP (activ. or repr. isof.)

A

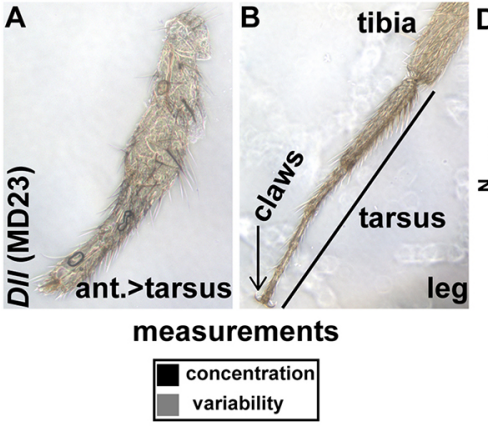

C

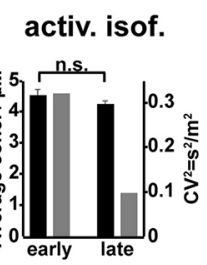

C'

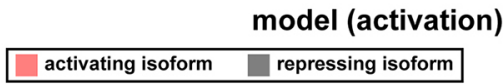

E

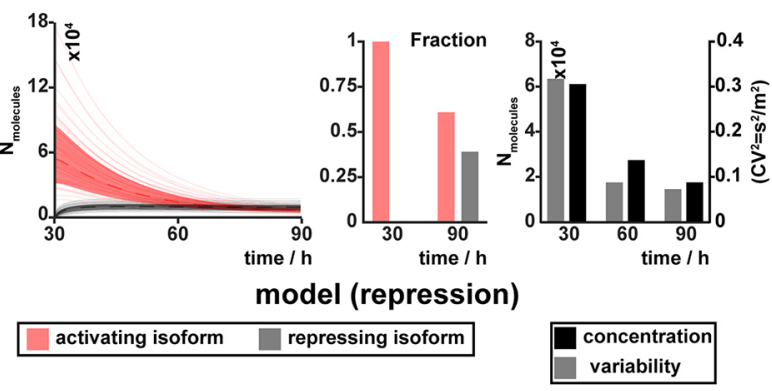

G

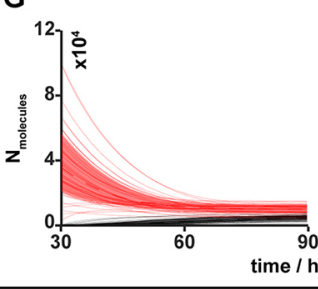

H Fraction I

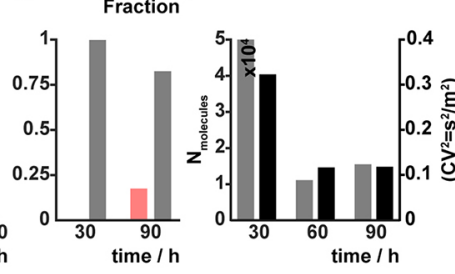

Perturbations (constit. repression by exogen. repressor)

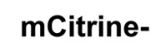
SynthScr
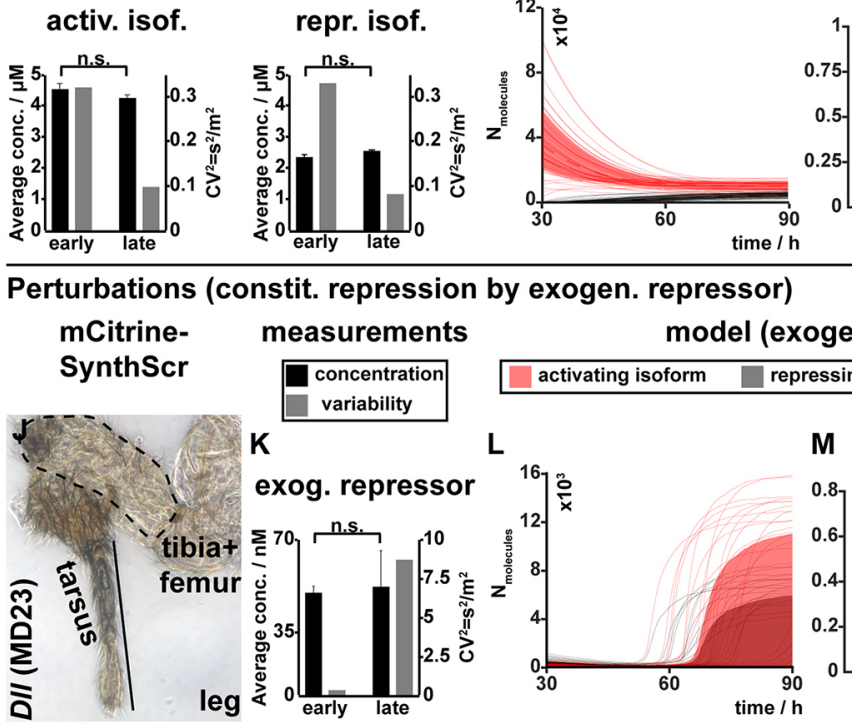

genous re

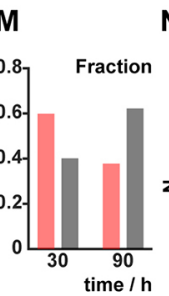

$\mathbf{N}$

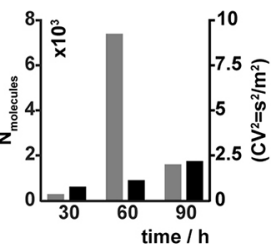

Fig. 6. Response of Antp to genetic perturbations. $(A, B)$ Overexpression of SynthAntp-eGFP long or short linker isoform result in tarsal transformations of the antenna (A), but normal leg development (B). These flies are fully viable and can be maintained as a stock. (C, $\left.\mathrm{C}^{\prime}\right)$ Antp concentration and variability, measured by FCS, in leg discs of second and third instar larvae upon SynthAntp-eGFP long or short linker isoform expression. Despite a persistent high concentration of Antp due to overexpression, variability is reduced. (D-I) Model response upon overexpression of Antp-activating or -repressing isoforms (similar to Fig. $5 \mathrm{H}-\mathrm{J}$ ). Trajectories of individual simulations are presented in $\mathrm{D}$ and $\mathrm{G}$ and the distribution of the Antp isoforms, predicted by the model, are presented in $\mathrm{E}$ and $\mathrm{H}$. (J) Overexpression of an exogenous repressor ( $\mathrm{Scr}$ ) results in abnormal distal leg development, bearing malformations of the tarsus and femur. (K-N) Similar to C-I (see also Fig. S14E-I'). Antp concentration and variability, measured by FCS in the proximal leg disc of second (early) and third (late) instar larvae upon overexpression of mCherry-SynthScr. observed differences in Antp concentration and cell-to-cell variability at early and late developmental stages.

\section{DISCUSSION}

In this work, we found that Antp auto-regulates its expression levels during development, starting from a preferentially auto-activating state early and transitioning to a preferentially auto-repressing state later. The early state is characterized by lower average Antp concentrations and high variability, whereas the opposite is true for the later repressing state. Without excluding other mechanisms, such as chromatin configuration, accessibility of Hox binding sites to Antp, the differential abundance of co-factors among developmental stages or different modes of interactions with different Antp isoforms, we have shown that differential expression of Antp isoforms is one contributing mechanism for the observed regulatory switch. These isoforms have preferentially activating or repressing activities on the Antp promoter, bind chromatin with different affinities and are themselves expressed in different relative amounts during development. A loss-of-function analysis of the isoforms in vivo will be required to provide a definitive answer on the relative contribution of the Antp isoform-mediated autoregulatory circuit towards the observed suppression of variability. CRISPR/Cas9-mediated genome manipulation, in principle, allows the generation of Antp loci that express only one or the other isoform. However, it is not clear whether these flies can reach the larval developmental stages, given the Antp embryonic functions and, in fact, strong biases towards only the activating or repressing isoform introduced by Antp-Gal4-mediated expression of either Antp isoform resulted in embryonic lethality. In the absence of such direct evidence, we turned to mathematical modelling and derived, based on our experimental data, a simple kinetic model of Antp auto-regulation that confirmed the plausibility of the proposed mechanism. In addition, the model generated predictions that could be verified by introducing genetic perturbations.

Negative auto-regulation has been identified as a frequently deployed mechanism for the reduction of noise (cell-to-cell variability) and the increase of regulatory robustness in various systems (Becskei and Serrano, 2000; Dublanche et al., 2006; Gronlund et al., 2013; Nevozhay et al., 2009; Shimoga et al., 2013; Thattai and van Oudenaarden, 2001). Auto-repression has been described for the Hox gene Ultrabithorax $(U b x)$ in haltere specification and as a mechanism of controlling Ubx levels against genetic variation (Crickmore et al., 2009; Garaulet et al., 2008), as well as in Ubx promoter regulation in Drosophila S2 cells (Krasnow et al., 1989). In contrast, an auto-activating mechanism is responsible for the maintenance of Deformed expression in the embryo (Kuziora and McGinnis, 1988). These experiments suggest similar mechanisms for establishing (auto-activation) or limiting (auto-repression) Hox TF levels and variability in different developmental contexts.

Our data suggest that the developmental switch from autoactivation to auto-repression is, at least in part, mediated by 
molecularly distinct Antp linker isoforms. Differences in affinities of different Hox TF isoforms, based on their linker between the YPWM motif and the homeodomain, have also been identified for the Hox TF Ubx. Interestingly, its linker is also subject to alternative splicing at the RNA level (Reed et al., 2010). In a similar way to Antp, the long linker Ubx isoform displays $20-25 \%$ of the affinity of DNA binding, when compared with the short linker isoforms, and the two isoforms are not functionally interchangeable in in vivo assays. Finally, the Ubx linker also affects the strength of its interaction with the Hox co-factor Extradenticle (Exd), underscoring the functional importance of linker length in Hox TF function (Saadaoui et al., 2011).

Mathematical modeling predicts that the Antp auto-regulatory circuit is robust with respect to initial conditions and extrinsic noise by suppressing cell-to-cell concentration variability even at high concentrations of any of the two Antp isoforms (auto-repressing or auto-activating). This 'buffering' capacity on cell-to-cell variability is reflected in the ability of flies to tolerate more than threefold overexpression of Antp without dramatic changes in endogenous Antp levels or generation of abnormal phenotypes. Therefore, two different isoforms produced from the same gene with opposing roles in transcriptional regulation and different auto-regulatory binding sites on the promoter of the gene seem to suffice to create a robust gene expression circuit that is able to 'buffer' perturbations of the starting conditions.

So far, we have only been able to indiscriminately increase or decrease Antp concentration at the tissue level and record the phenotypic outcome of these boundary states. It will be interesting to test whether controlled perturbations of TF variability at the tissue level that render TF concentration patterns less, or more, noisy among neighboring cells, while maintaining similar mean protein concentrations, lead to abnormal phenotypes. The technology to selectively manipulate expression variability of specific TFs in a developing tissue is yet to be established.

\section{MATERIALS AND METHODS \\ Fly stocks used}

The Antp-eGFP MiMIC line was a kind gift from Hugo J. Bellen (HHMI Baylor College of Medicine, Duncan Neurological Research Institute Houston, TX, USA; Bloomington Drosophila Stock Center, 59790). The atonal (VDRC ID 318959), brinker (VDRC ID 318246), spalt major (VDRC ID 318068), yorkie (VDRC ID 318237), senseless (VDRC ID 318017) and Sex combs reduced (VDRC ID 318441) fosmid lines are available from the Vienna Drosophila Resource Center (VDRC) and were generated recently in our laboratory (Sarov et al., 2016). The fork head (stock 43951), grainy head (stock 42272), Abdominal B (stock 38625), eyeless, (stock 42271), spineless (transcript variant A, stock 42289) and grain (stock 58483) tagged BACs were generated by Rebecca Spokony and Kevin P. White (Department of Human Genetics, The University of Chicago, IL, USA) and are available at the Bloomington Stock Center. For the scalloped gene, a GFP-trap line was used (Buszczak et al., 2007), a kind gift from Allan C. Spradling's laboratory (line CA07575), with which genome-wide chromatin immunoprecipitation experiments have been performed (Slattery et al., 2013). For the spineless gene, Bloomington stock 42676, which tags isoforms C and D of the Spineless protein, has been also tried in fluorescence imaging and FCS experiments, but did not yield detectable fluorescence in the antennal disc, rendering it inappropriate for use in our analysis. Therefore, we used stock 42289, which tags the A isoform of theprotein. For the eyeless gene, the FlyFos015860(pRedFlpHgr)(ey13630::2XTY1-SGFP-V5-preTEV-BLRP-3XFLAG)dFRT line (VDRC ID 318018) has been tried also in fluorescence imaging and FCS experiments, but did not yield detectable fluorescence in the eye disc for it to be used in our analysis. The act5C-FRT-yellow-FRT-Gal4 ( $A y$-Gal4) line used for clonal overexpression or RNAi knockdown has been described (Ito et al., 1997). The UAS-Antp lines (synthetic and full-length), as well as UAS-SynthScr constructs, have been previously described (Papadopoulos et al., 2011, 2010). The Dll-Gal4 (MD23) line was a kind gift from Ginés Morata (CBMSO, Universidad Autónoma de Madrid, Spain) (Calleja et al., 1996). 69B-Gal4 and ptc-Gal4 were obtained from the Bloomington Stock Center. The Antp P1-lacZ and P2-lacZ have been previously described (Engstrom et al., 1992; Zink et al., 1991). The P1 reporter construct spans the region between $9.4 \mathrm{~kb}$ upstream of the P1 promoter transcription initiation site and $7.8 \mathrm{~kb}$ downstream into the first intron, including the first exon sequences and thus comprising $17.2 \mathrm{~kb}$ of Antp regulatory sequences (pAPT 1.8). The line used was an insertion of the pAPT 1.8 vector bearing the $\mathrm{P} 1$ promoter regulatory sequences upstream of an actin-lac $Z$ cytoplasmic reporter and was inserted in cytogenetic location $99 \mathrm{~F}$ on the right chromosomal arm of chromosome 3. The Antp-RNAi line was from VDRC, line KK101774. UAS-eGFP stock was a kind gift from Konrad Basler (Universität Zürich, Switzerland). We are indebted to Sebastian Dunst (Max-Planck Institute of Molecular Cell Biology and Genetics, Dresden, Germany) for generating the ubi-FRT-mCherry(stop)-FRTGal4(VK37)/CyO line, which drives clonal overexpression upon flippase excision, while simultaneously marking cells by the loss of mCherry. For red-color labeling of clones the act5C-FRT-CD2-FRT-Gal4, UAS-mRFPI (NLS)/TM3 stock 30558 from the Bloomington Stock Center was used. For marking the ectopic expression domain of untagged Antp proteins, the UAS- $m R F P 1$ (NLS)/TM3 stock 31417 from the Bloomington Stock Center was used. The MS243-Gal4; UAS-GFP/CyO line was a kind gift from the laboratory of Ernesto Sánchez-Herrero (CBMSO, Universidad Autónoma de Madrid, Spain).

\section{Fly genotypes corresponding to fluorescence images}

Fig. 2B,B': $h s$-flp/+; act5C-FRT-yellow-FRT-Gal4/+; UAS-SynthAntp long linker-eGFP/+

Fig. 2C, C': hs-flp/+; act5C-FRT-yellow-FRT-Gal4, UAS-eGFP/+; UASAntp long linker (full-length, untagged)/+

Fig. 2G,G': Dll-Gal4 (MD23)/+; UAS-SynthAntp-eGFP/Antp P1-lacZ

Fig. 3B, $\mathrm{B}^{\prime}, \mathrm{G}^{\prime}, \mathrm{G}^{\prime}$ : $h s$-flp/+; ubi-FRT-mChery-FRT-Gal4/+; Antp-eGFP

(MiMIC)/UAS-Antp long linker (full-length, untagged)

Fig. 3C, $\mathrm{C}^{\prime}: h s$-flp/+; UAS-Antp ${ }^{R N A i} /+$; Antp P1-lacZ/act5C-FRT-CD2FRT-Gal4, UAS-mRFP1(NLS)

Fig. $3 \mathrm{H}, \mathrm{H}^{\prime}: h s$-flp/+; UAS-Antp ${ }^{R N A i} /$ act $5 C$-FRT-yellow-FRT-Gal4, UASeGFP; Antp P1-lacZ/+

Fig. 4B,B': ptc-Gal4/+; UAS-SynthAntp long linker-eGFP/+

Fig. 4C,C': Dll-Gal4 (MD23)/+; UAS-SynthAntp long linker-eGFP/Antp $\mathrm{P} 1-\mathrm{lac} Z$

Fig. 4F, $\mathrm{F}^{\prime}$ : ptc-Gal4/+; UAS-SynthAntp long linker-eGFP/+

Fig. 4G,G': Dll-Gal4 (MD23)/+; UAS-SynthAntp short linker-eGFP/Antp P1-lac Z

Fig. 5A: Dll-Gal4 (MD23)/+; UAS-SynthAntp long linker-eGFP/+

Fig. 5B: ptc-Gal4/+; UAS-SynthAntp long linker-eGFP/+

Fig. 5C: Dll-Gal4 (MD713)/+; UAS-SynthAntp long linker-eGFP/+ Fig. 5D: 69B-Gal4/UAS-SynthAntp long linker-eGFP

Fig. 6A,B: Dll-Gal4 (MD23)/+; UAS-SynthAntp long linker-eGFP/+ or Dll-Gal4 (MD23)/+; UAS-SynthAntp short linker-eGFP/+

Fig. 6J: Dll-Gal4 (MD23)/+; UAS-mCitrine-SynthScr/+

\section{Preparation of second and third instar imaginal discs for FCS} measurements

For FCS measurements, imaginal discs (eye-antennal, wing, leg, humeral and genital) and salivary glands were dissected from third instar wandering larvae, or wing and leg discs from second instar larvae, in Grace's insect tissue culture medium (Thermo Fisher Scientific, 11595030) and transferred to an eight-well chambered coverglass (Nunc Lab-Tek, 155411) containing PBS just prior to imaging or FCS measurements. Floating imaginal discs or salivary glands were sunk to the bottom of the well using forceps.

\section{Immunostainings in larval imaginal discs}

Larval imaginal discs were stained according to Papadopoulos et al. (2010). Staining for the endogenous Antp protein were performed using a mouse anti-Antp antibody (Developmental Studies Hybridoma Bank, University of 
Iowa, anti-Antp 4C3) in a dilution of 1:250 for embryos and 1:500 for imaginal discs. eGFP, or eGFP-tagged proteins, were stained using mouse or rabbit anti-GFP antibodies from Thermo Fisher Scientific at 1:500 in imaginal discs and 1:250 in embryos. mRFP1 was stained using a Chromotek rat anti-RFP antibody. For Antp P1 promoter staining in imaginal discs, we used the mouse anti- $\beta$-galactosidase $40-1 \mathrm{a}$ antibody from Developmental Studies Hybridoma Bank, University of Iowa at 1:50. The rabbit anti-Scr antibody was used at 1:300 (LeMotte et al., 1989). Confocal images of antibody staining represent predominantly $z$-projections and Zeiss LSM510, Zeiss LSM700 or Zeiss LSM880 Airyscan confocal laserscanning microscopy systems with an inverted stand Axio Observer microscope were used for imaging. Image processing and quantifications have been performed in Fiji (Schindelin et al., 2012). For optimal spectral separation, secondary antibodies coupled to Alexa405, Alexa488, Alexa594 and Cy5 (Thermo Fisher Scientific) were used.

\section{Colocalization of wild-type and eGFP-tagged MiMIC Antp alleles in imaginal discs}

To examine whether the pattern of the MiMIC Antp-eGFP fusion protein recapitulates the Antp wild-type expression pattern in both embryo and larval imaginal discs, we performed immunostaining of heterozygous Antp$e G F P$ and wild-type flies to visualize the embryonic (stage 13) and larval expression of Antp and eGFP. In this experiment, we (1) visualized the overlap between eGFP and Antp (the eGFP pattern reflects the protein encoded by the MiMIC allele, whereas the Antp pattern reflects the sum of protein produced by the MiMIC allele and the allele of the balancer chromosome); and (2) compared the eGFP expression pattern to the Antp expression pattern in wild-type discs and embryos.

\section{Induction of early and late overexpression and RNAi-knockdown clones in imaginal discs}

Genetic crosses with $~ 100$ virgin female and 100 male flies were set up in bottles and the flies were allowed to mate for 2 days. Then, they were transferred to new bottles and embryos were collected for $6 \mathrm{~h}$ at $25^{\circ} \mathrm{C}$. Flies were then transferred to fresh bottles and kept until the next collection at $18^{\circ} \mathrm{C}$. To asses Antp auto-activation, the collected eggs were allowed to grow at $25^{\circ} \mathrm{C}$ for $26 \mathrm{~h}$ from the midpoint of collection, when they were subjected to heat-shock by submersion of the bottles in a water bath at $38^{\circ} \mathrm{C}$ for $30 \mathrm{~min}$ and then placed back at $25^{\circ} \mathrm{C}$ until they reached the stage of third instar wandering larvae, when they were collected for dissection, fixation and staining with antibodies. To assess Antp auto-repression, the same procedure was followed, except that the heat-shock was performed at $60 \mathrm{~h}$ of development after the midpoint of embryo collection. Whenever necessary, larval genotypes were selected under a dissection stereomicroscope with green and red fluorescence filters on the basis of deformed (dfd)-YFP bearing balancer chromosomes (Le et al., 2006) and visual inspection of fluorescence in imaginal discs.

\section{Measurement of Antp transcript variant abundance}

The linker between the Antp YPWM motif and the homeodomain contains the sequence RSQFGKCQE. Short linker isoforms encode the sequence RSQFE, whereas long linker isoforms are generated by alternative splicing of a 12 base pair sequence encoding the four amino acid sequence GKCQ into the mRNA. We initially designed primer pairs for RT-qPCR experiments to distinguish between the short and long linker mRNA variants. For the short linker variant, we used nucleotide sequences corresponding to RSQFERKR (with RKR being the first 3 amino acids of the homeodomain). For detection of the long linker variant, we designed primers either corresponding to the RSQFGKCQ sequence or to GKCQERKR. We observed in control PCRs (using plasmid DNA harboring either a long or a short linker cDNA) that primers designed for the short linker variant still amplified the long linker one. Moreover, with linker sequences differing in only four amino acids, encoded by 12 base pairs, primer pairs flanking the linker could also not be used, because, owing to very similar sizes, both variants would be amplified in RTqPCR experiments with almost equal efficiencies. Therefore, we used primer pairs flanking the linker region to indiscriminately amplify short and long linker variants, using non-saturating PCR (18 cycles) on total cDNA generated from total RNA. We then resolved and assessed the relative amounts of long and short linker amplicons in a second step using Fragment Analyzer (Advanced Analytical). RNA was extracted from stage 13 embryos, second instar larvae at $60 \mathrm{~h}$ of development, and leg or wing discs from third instar wandering larvae using the Trizol reagent (Thermo Fisher Scientific), following the manufacturer's instructions. Total RNA amounts were measured using NanoDrop and equal amounts were used to synthesize cDNA using the High-Capacity RNA-to-cDNA Kit (Thermo Fisher Scientific), following the manufacturer's instructions. Total cDNA yields were measured by NanoDrop and equal amounts were used in PCR, using inhouse produced Taq polymerase. Plasmid DNA (10 ng), bearing either a long or a short transcript cDNA were used as a control. PCR product abundance was analyzed both by agarose gel electrophoresis and using Fragment Analyzer.

The quantification of the transcript variant concentration (Fig. 4D,H) was made by considering $100 \%$ (value equal to 1 on the $y$-axis) as the sum of long and short isoforms at each developmental stage, whereas the quantification of the relative activation and repression efficiency was performed considering the short linker variant as having $100 \%$ repression and the long linker variant as having $100 \%$ activation efficiency (values equal to 1 on the $y$-axis).

\section{Quantification of the relative repressing and activating efficiencies of different Antp isoforms}

Quantification of the relative efficiency of Antp activating and repressing isoforms (Fig. 4D,H) were performed in Fiji (Schindelin et al., 2012) by outlining the total region of repression or activation of Antp protein or P1 reporter staining and quantification of the relative fluorescence intensity of the selected regions. From the calculated values, we have subtracted the values obtained by outlining and calculating Antp protein or reporter $\beta$-galactosidase staining background in the region of expression of an eGFPencoding transgene alone (negative control). Five to seven imaginal disc images per investigated genotype were used for analysis. For the repression assay, the obtained values have been normalized over the intensity of Antp protein calculated in the region of overlap between an eGFP-expressing transgene and Antp (negative control). In both cases (repression and activation), the highest efficiencies per transcript variant (for repression, the short linker isoform; for activation the long linker isoform) have been set to $100 \%$.

\section{Fluorescence microscopy imaging of live imaginal discs and FCS}

Fluorescence imaging and FCS measurements were performed on two uniquely modified confocal laser scanning microscopy systems, both featuring the ConfoCor3 system (Zeiss) and consisting of either an inverted microscope for transmitted light and epifluorescence (Axiovert $200 \mathrm{M}$ ); a VIS-laser module comprising the $\mathrm{Ar} / \mathrm{ArKr}$ (458, 477, 488 and $514 \mathrm{~nm}$ ), $\mathrm{HeNe} 543 \mathrm{~nm}$ and HeNe $633 \mathrm{~nm}$ lasers and the scanning module LSM510 META; or a Zeiss LSM780 inverted setup, comprising Diode $405 \mathrm{~nm}$, Ar multiline 458, 488 and $514 \mathrm{~nm}$, DPSS $561 \mathrm{~nm}$ and HeNe $633 \mathrm{~nm}$ lasers. Both instruments were modified to enable detection using silicon Avalanche Photo Detectors (SPCM-AQR-1X; PerkinElmer) for imaging and FCS (Vukojevic et al., 2008). Images were recorded at a $512 \times 512$ pixel resolution. C-Apochromat 40×/1.2 W UV-VIS-IR objectives were used throughout. Fluorescence intensity fluctuations were recorded in arrays of 10 consecutive measurements, each measurement lasting $10 \mathrm{~s}$. Averaged curves were analyzed using the software for online data analysis or exported and fitted offline using the OriginPro 8 data analysis software (OriginLab). In both cases, the nonlinear least square fitting of the autocorrelation curve was performed using the Levenberg-Marquardt algorithm. Quality of the fitting was evaluated by visual inspection and by residuals analysis. Control FCS measurements to assess the detection volume were routinely performed prior to data acquisition, using dilute solutions of known concentration of Rhodamine $6 \mathrm{G}$ and Alexa488 dyes. The variability between independent measurements reflects variabilities between cells, rather than imprecision of FCS measurements. For more details on fluorescence microscopy imaging and FCS, refer to the supplementary Materials and Methods.

In Fig. 1A-H the workflow of FCS measurements is schematically represented. Live imaging of imaginal discs, expressing endogenously tagged TFs, visualized by fluorescence microscopy, and neighboring cells, expressing 
TFs at different levels, selected for FCS measurements (Fig. 1A,B). FCS measurements were performed by focusing the laser light into the nucleus (Fig. 1C,D) and recording fluorescence intensity fluctuations (Fig. 1E), which are generated by TF molecules quickly/slowly diffusing into/out of the confocal detection volume (Fig. 1D). The recorded fluctuations are subjected to temporal autocorrelation analysis, which generates temporal autocorrelation curves (henceforth referred to as FCS curves) that, by fitting with an appropriate model function (see supplementary Materials and Methods), yield information about the absolute concentration of fluorescent molecules (Fig. 1F) and their corresponding diffusion times, as well as the fraction of fast- and slowly-diffusing TF molecules. Differences in diffusion and the fractions of faster- and slower-diffusing molecules can be readily visualized after normalization to the same amplitude (Fig. 1G). The concentration of molecules is inversely proportional to the $y$-axis amplitude at the zero lag time, i.e. the origin of the FCS curve (Fig. 1F). FCS curves normalized to the same amplitude clearly show a shift of the FCS curves to longer lag times when processes that slow down the diffusion of TF molecules, such as binding to very large molecules (e.g. chromosomal DNA), are present (Fig. 1G). Measurements in a collection of neighboring cell nuclei also allow the calculation of protein concentration variability at the live tissue level (Fig. 1H)

\section{Sample size, biological and technical replicates}

For the measurement of TF molecular numbers and variability (Fig. 1 and Fig. S1), seven to ten larvae of each fly strain were dissected, yielding at least 15 imaginal discs, which were used in FCS analysis. For the Fkh TF, seven pairs of salivary glands were analyzed and for $\mathrm{AbdB}, 12$ genital discs were dissected from 12 larvae. More than 50 FCS measurements were performed in patches of neighboring cells of these dissected discs, in the regions of expression indicated in Fig. S1 by arrows. Imaginal discs from the same fly strain (expressing a given endogenously tagged TF) were analyzed on at least three independent occasions (FCS sessions), taking place on different days (biological replicates). For Antp, which was further analyzed in this study, more than 20 independent FCS sessions were used. As routinely carried out with FCS measurements in live cells, these measurements were evaluated during acquisition and subsequent analysis, and, based on their quality (high counts per molecule and low photobleaching), were included in the calculation of concentration and variability. In Fig. S1Q, $n$ denotes the number of FCS measurements included in the calculations.

For experiments involving immunostaining in imaginal discs to investigate the auto-regulatory behavior of Antp (Figs 2-5, except for the temporally resolved auto-activating and repressing study of Antp in Fig. 3, as discussed above), 14-20 male and female flies were mated in bottles and 10 larvae were selected by means of fluorescent balancers and processed downstream. Up to 20 imaginal discs were visualized by fluorescence microscopy and highresolution $z$-stacks were acquired for three to five representative discs or disc regions of interest per experiment. All experiments were performed in triplicate, except for the temporal analysis of Antp auto-regulatory behavior in Fig. 3, which was performed six times, and the quantification of repression efficiency of short and long linker Antp isoforms in Fig. 4, which was performed five times. For the quantification of transcript variant abundance in Fig. 4D,H, RNA and, thus, cDNA were prepared from each stage three independent times (biological replicates) and the transcript abundance per RNA/cDNA sample was also analyzed three times.

For the experiments involving perturbations in Antp expression, during which the proper development of the leg and the notum were assessed in Fig. 5, more than 100 adult flies were analyzed and this experiment was performed more than 10 times independently.

\section{Statistical significance}

Fig. 2D: Statistical significance was determined using a two-tailed Student's $t$-test $[* * * P<0.001$ and $* P<0.05$, namely $P$ (repression clone vs surrounding Antp protein) $=1.36 \times 10^{-15}$ and $P$ (repression clone vs surrounding Antp reporter) $\left.=3.17 \times 10^{-16}\right]$.

Fig. 2H: Statistical significance was determined using a two-tailed Student's $t$-test $[* * * P<0.001$ and $* P<0.05$, namely $P(D l l$ expression domain vs surrounding Antp reporter) $\left.=1.55 \times 10^{-17}\right]$.

Fig. 3E: Statistical significance was determined using a two-tailed Student's $t$-test $\{* * * P<0.001$ and $* P<0.05$, namely $P$ (early activation clone vs surrounding Antp protein) $=6.23 \times 10^{-13}$ and $P$ [early knockdown (RNAi) clone vs surrounding Antp reporter] $\left.=2.98 \times 10^{-9}\right\}$.

Fig. 3F: Statistical significance was determined using a two-tailed Student's $t$-test $\left[* * * P<0.001\right.$ and $* P<0.05$, namely $P\left(2\right.$ nd vs 3 rd instar $\left.\tau_{\mathrm{D} 2}\right)=$ $7.2 \times 10^{-4}, P\left(2 \mathrm{nd}\right.$ vs $3 \mathrm{rd}$ instar $\left.\tau_{\mathrm{D} 2}\right)=7.2 \times 10^{-4}$ and $P(2$ nd vs 3 rd instar variation) $=3.4 \times 10^{-2}$.

Fig. 3K: Statistical significance was determined using a two-tailed Student's $t$-test $\{* * * P<0.001$ and $* P<0.05$, namely $P$ (late repression clone vs surrounding Antp protein) $=3.98 \times 10^{-17}$ and $P$ [late knockdown (RNAi) clone vs surrounding Antp reporter $\left.]=1.16 \times 10^{-21}\right\}$.

Fig. 4D,H: Statistical significance was determined using a two-tailed Student's $t$-test between measurements performed with the long linker (auto-activating) isoform (Fig. 4D) and the short linker (auto-repressing) isoform (Fig. 4H) $\{* * * P<0.001$ and $* P<0.05$, namely $P$ (embryo long vs short concentration $)=3.16 \times 10^{-5}, P(2 \mathrm{nd}$ instar long vs short concentration $)=1.16 \times 10^{-4}, P($ long vs short relative activation $)=$ $4.1 \times 10^{-3}, P($ long vs short relative activation $)=4.1 \times 10^{-3}, P($ long vs short relative repression $)=2.4 \times 10^{-4}$ and $P$ [long vs short DNA-bound fraction $\left.(\mathrm{FCS})]=5.6 \times 10^{-10}\right\}$.

Fig. 5E: Statistical significance was determined using a two-tailed Student's $t$-test $\left[* * * P<0.001\right.$, namely $P(M D 23$ vs $p t c)=3.54 \times 10^{-4}$ and $P(M D 713$ vs $69 B)=4.15 \times 10^{-9}$ ].

Fig. 6C-C': Statistical significance was determined using a two-tailed Student's $t$-test $P$ (early vs late conc. leg disc, o/e activator) $=0.679$ and $P$ (early vs late conc. leg disc, o/e repressor) $=0.454$.

Fig. 6K: Statistical significance was determined using a two-tailed Student's $t$-test $P$ (early vs late conc. leg disc, o/e exog. repr.) $=0.892$.

\section{Acknowledgements}

We are deeply saddened by the unexpected passing of Prof. Walter J. Gehring at the very inception of this work, when the project was still in the planning and preliminary data gathering stage. Prof. Gehring was an extraordinary human being and a scientific giant, whose work will continue to educate and inspire generations to come. D.K.P. expresses his gratitude to P.T. for outstanding scientific, and uninterrupted financial, support. D.K.P. acknowledges Markus Burkhardt, Sylke Winkler, Aliona Bogdanova and the Light Microscopy facility of MPI-CBG. D.K.P. is also grateful to Konstantinos Papadopoulos for advice on the mathematical analysis. The authors thank Wendy Bickmore, Jan Brugues and Thomas M. Schultheiss for critical comments on the manuscript.

\section{Competing interests}

The authors declare no competing or financial interests.

\section{Author contributions}

Conceptualization: D.K.P., C.Z., P.T.; Methodology: D.K.P., K.S., Y.E., C.Z., V.V., P.T.; Software: D.K.P., C.Z.; Validation: D.K.P., C.Z., V.V.; Formal analysis: D.K.P., K.S., C.Z., V.V.; Investigation: D.K.P.; Resources: D.K.P., Y.E., R.R., V.V., P.T.; Writing - original draft: D.K.P., V.V., P.T.; Writing - review \& editing: D.K.P., K.S., Y.E., L.T., R.R., C.Z., V.V., P.T.; Visualization: D.K.P.; Supervision: D.K.P., P.T.; Project administration: D.K.P.; Funding acquisition: D.K.P., P.T.

\section{Funding}

D.K.P. was supported by a long-term fellowship from the Schweizerischer Nationalfonds zur Förderung der Wissenschaftlichen Forschung (PBBSP-138700), by a long-term fellowship from the Federation of European Biochemical Societies, by the Max-Planck-Gesellschaft (through funds to P.T.) and by a Chancellor's Fellowship from the University of Edinburgh. V.V. was supported by the Knut och Alice Wallenbergs Stiftelse and by Karolinska Institutet research funds. Deposited in PMC for immediate release.

\section{Supplementary information}

Supplementary information available online at

http://dev.biologists.org/lookup/doi/10.1242/dev.168179.supplemental

\section{References}

Abbott, M. K. and Kaufman, T. C. (1986). The relationship between the functional complexity and the molecular organization of the Antennapedia locus of Drosophila melanogaster. Genetics 114, 919-942.

Baumgartner, S., Littleton, J. T., Broadie, K., Bhat, M. A., Harbecke, R., Lengyel, J. A., Chiquet-Ehrismann, R., Prokop, A. and Bellen, H. J. (1996). A Drosophila neurexin is required for septate junction and blood-nerve barrier formation and function. Cell 87, 1059-1068. 
Becskei, A. and Serrano, L. (2000). Engineering stability in gene networks by autoregulation. Nature 405, 590-593.

Blake, W. J., KAErn, M., Cantor, C. R. and Collins, J. J. (2003). Noise in eukaryotic gene expression. Nature 422, 633-637.

Boettiger, A. N. and Levine, M. (2013). Rapid transcription fosters coordinate snail expression in the Drosophila embryo. Cell Reports 3, 8-15.

Buszczak, M., Paterno, S., Lighthouse, D., Bachman, J., Planck, J., Owen, S., Skora, A. D., Nystul, T. G., Ohlstein, B., Allen, A. et al. (2007). The carnegie protein trap library: a versatile tool for Drosophila developmental studies. Genetics 175, 1505-1531

Calleja, M., Moreno, E., Pelaz, S. and Morata, G. (1996). Visualization of gene expression in living adult Drosophila. Science 274, 252-255.

Clark, N. M., Hinde, E., Winter, C. M., Fisher, A. P., Crosti, G., Blilou, I., Gratton, E., Benfey, P. N. and Sozzani, R. (2016). Tracking transcription factor mobility and interaction in Arabidopsis roots with fluorescence correlation spectroscopy eLife 5, e14770.

Crickmore, M. A., Ranade, V. and Mann, R. S. (2009). Regulation of Ubx expression by epigenetic enhancer silencing in response to Ubx levels and genetic variation. PLoS Genet. 5, e1000633.

Dublanche, Y., Michalodimitrakis, K., Kümmerer, N., Foglierini, M. and Serrano, L. (2006). Noise in transcription negative feedback loops: simulation and experimental analysis. Mol. Syst. Biol. 2, 41.

Dworkin, I., Lee, W., McCloskey, F. and Larsen, E. (2007). Complex genetic interactions govern the temporal effects of Antennapedia on antenna-to-leg transformations in Drosophila melanogaster. J. Genet. 86, 111-123.

Elowitz, M. B., Levine, A. J., Siggia, E. D. and Swain, P. S. (2002). Stochastic gene expression in a single cell. Science $\mathbf{2 9 7}, 1183-1186$

Emerald, B. S. and Cohen, S. M. (2004). Spatial and temporal regulation of the homeotic selector gene Antennapedia is required for the establishment of leg identity in Drosophila. Dev. Biol. 267, 462-472.

Engstrom, Y., Schneuwly, S. and Gehring, W. J. (1992). Spatial and tempora expression of an antennapedia lac $Z$ gene construct integrated into the endogenous antennapedia gene of drosophila-melanogaster. Dev. Genes Evol. 201, 65-80.

Franz, K., Singh, A. and Weinberger, L. S. (2011). Lentiviral vectors to study stochastic noise in gene expression. Methods Enzymol. 497, 603-622.

Garaulet, D. L., Foronda, D., Calleja, M. and Sanchez-Herrero, E. (2008) Polycomb-dependent Ultrabithorax Hox gene silencing induced by high Ultrabithorax levels in Drosophila. Development 135, 3219-3228.

Gillespie, D. T. (2007). Stochastic simulation of chemical kinetics. Annu. Rev. Phys. Chem. 58, 35-55

Gregor, T., Tank, D. W., Wieschaus, E. F. and Bialek, W. (2007a). Probing the limits to positional information. Cell 130, 153-164.

Gregor, T., Wieschaus, E. F., McGregor, A. P., Bialek, W. and Tank, D. W (2007b). Stability and nuclear dynamics of the bicoid morphogen gradient. Cell 130, 141-152.

Gronlund, A., Lotstedt, P. and Elf, J. (2013). Transcription factor binding kinetics constrain noise suppression via negative feedback. Nat. Commun. 4, 1864.

Halford, S. E. and Marko, J. F. (2004). How do site-specific DNA-binding proteins find their targets? Nucleic Acids Res. 32, 3040-3052.

Holloway, D. M. and Spirov, A. V. (2015). Mid-embryo patterning and precision in Drosophila segmentation: Kruppel dual regulation of hunchback. PLOS ONE 10, e0118450.

Holloway, D. M., Lopes, F. J. P., da Fontoura Costa, L., Travençolo, B. A. N. Golyandina, N., Usevich, K. and Spirov, A. V. (2011). Gene expression noise in spatial patterning: hunchback promoter structure affects noise amplitude and distribution in Drosophila segmentation. PLoS Comput. Biol. 7, e1001069.

Ito, K., Awano, W., Suzuki, K., Hiromi, Y. and Yamamoto, D. (1997). The Drosophila mushroom body is a quadruple structure of clonal units each of which contains a virtually identical set of neurones and glial cells. Development 124 761-771.

Jorgensen, E. M. and Garber, R. L. (1987). Function and misfunction of the two promoters of the Drosophila Antennapedia gene. Genes Dev. 1, 544-555.

Kang, M., Piliszek, A., Artus, J. and Hadjantonakis, A.-K. (2013). FGF4 is required for lineage restriction and salt-and-pepper distribution of primitive endoderm factors but not their initial expression in the mouse. Development 140, 267-279.

Kaur, G., Costa, M. W., Nefzger, C. M., Silva, J., Fierro-González, J. C., Polo, J. M., Bell, T. D. M. and Plachta, N. (2013). Probing transcription factor diffusion dynamics in the living mammalian embryo with photoactivatable fluorescence correlation spectroscopy. Nat. Commun. 4, 1637.

Kelso, R. J., Buszczak, M., Quinones, A. T., Castiblanco, C., Mazzalupo, S. and Cooley, L. (2004). Flytrap, a database documenting a GFP protein-trap insertion screen in Drosophila melanogaster. Nucleic Acids Res. 32, D418-D420.

Krasnow, M. A., Saffman, E. E., Kornfeld, K. and Hogness, D. S. (1989) Transcriptional activation and repression by Ultrabithorax proteins in cultured Drosophila cells. Cell 57, 1031-1043

Kuziora, M. A. and McGinnis, W. (1988). Autoregulation of a Drosophila homeotic selector gene. Cell 55, 477-485

Lam, C. S., Mistri, T. K., Foo, Y. H., Sudhaharan, T., Gan, H. T., Rodda, D., Lim L. H., Chou, C., Robson, P., Wohland, T. et al. (2012). DNA-dependent Oct4-
Sox2 interaction and diffusion properties characteristic of the pluripotent cell state revealed by fluorescence spectroscopy. Biochem. J. 448, 21-33.

Le, T., Liang, Z., Patel, H., Yu, M. H., Sivasubramaniam, G., Slovitt, M. Tanentzapf, G., Mohanty, N., Paul, S. M., Wu, V. M. et al. (2006). A new family of Drosophila balancer chromosomes with a w- dfd-GMR yellow fluorescent protein marker. Genetics 174, 2255-2257.

LeMotte, P. K., Kuroiwa, A., Fessler, L. I. and Gehring, W. J. (1989). The homeotic gene Sex Combs Reduced of Drosophila: gene structure and embryonic expression. EMBO J. 8, 219-227.

Little, S. C., Tikhonov, M. and Gregor, T. (2013). Precise developmental gene expression arises from globally stochastic transcriptional activity. Cell 154, 789-800.

Lo, C.-A., Kays, I., Emran, F., Lin, T.-J., Cvetkovska, V. and Chen, B. E. (2015) Quantification of protein levels in single living cells. Cell Reports 13, 2634-2644.

Lucas, T., Ferraro, T., Roelens, B., De Las Heras Chanes, J., Walczak, A. M. Coppey, M. and Dostatni, N. (2013). Live imaging of bicoid-dependent transcription in Drosophila embryos. Curr. Biol. 23, 2135-2139.

Mistri, T. K., Devasia, A. G., Chu, L. T., Ng, W. P., Halbritter, F., Colby, D. Martynoga, B., Tomlinson, S. R., Chambers, I., Robson, P. et al. (2015) Selective influence of Sox2 on POU transcription factor binding in embryonic and neural stem cells. EMBO Rep. 16, 1177-1191.

Morin, X., Daneman, R., Zavortink, M. and Chia, W. (2001). A protein trap strategy to detect GFP-tagged proteins expressed from their endogenous loci in Drosophila. Proc. Natl. Acad. Sci. USA 98, 15050-15055

Muller, C. B., Loman, A., Pacheco, V., Koberling, F., Willbold, D., Richtering, W. and Enderlein, J. (2008). Precise measurement of diffusion by multi-color dualfocus fluorescence correlation spectroscopy. Epl-Europhys Lett. 83

Nagarkar-Jaiswal, S., Lee, P. T., Campbell, M. E., Chen, K., Anguiano-Zarate, S. Gutierrez, M. C., Busby, T., Lin, W. W., He, Y., Schulze, K. L. et al. (2015). A library of MiMICs allows tagging of genes and reversible, spatial and temporal knockdown of proteins in Drosophila. eLife 4, e05338.

Nevozhay, D., Adams, R. M., Murphy, K. F., Josic, K. and Balazsi, G. (2009) Negative autoregulation linearizes the dose-response and suppresses the heterogeneity of gene expression. Proc. Natl. Acad. Sci. USA 106, 5123-5128.

Newman, J. R., Ghaemmaghami, S., Ihmels, J., Breslow, D. K., Noble, M. DeRisi, J. L. and Weissman, J. S. (2006). Single-cell proteomic analysis of S. cerevisiae reveals the architecture of biological noise. Nature 441, 840-846.

Papadopoulos, D. K., Vukojevic, V., Adachi, Y., Terenius, L., Rigler, R. and Gehring, W. J. (2010). Function and specificity of synthetic Hox transcription factors in vivo. Proc. Natl. Acad. Sci. USA 107, 4087-4092.

Papadopoulos, D. K., Resendez-Perez, D., Cardenas-Chavez, D. L., VillanuevaSegura, K., Canales-del-Castillo, R., Felix, D. A., Funfschilling, R. and Gehring, W. J. (2011). Functional synthetic Antennapedia genes and the dua roles of YPWM motif and linker size in transcriptional activation and repression. Proc. Natl. Acad. Sci. USA 108, 11959-11964.

Papadopoulos, D. K., Krmpot, A. J., Nikolic, S. N., Krautz, R., Terenius, L., Tomancak, P., Rigler, R., Gehring, W. J. and Vukojevic, V. (2015). Probing the kinetic landscape of Hox transcription factor-DNA binding in live cells by massively parallel Fluorescence Correlation Spectroscopy. Mech. Dev. 138, 218-225.

Paré, A., Lemons, D., Kosman, D., Beaver, W., Freund, Y. and McGinnis, W. (2009). Visualization of individual Scr mRNAs during Drosophila embryogenesis yields evidence for transcriptional bursting. Curr. Biol. 19, 2037-2042.

Perez-Camps, M., Tian, J., Chng, S. C., Sem, K. P., Sudhaharan, T., Teh, C. Wachsmuth, M., Korzh, V., Ahmed, S. and Reversade, B. (2016). Quantitative imaging reveals real-time Pou5f3-Nanog complexes driving dorsoventra mesendoderm patterning in zebrafish. eLife 5, e11475.

Plachta, N., Bollenbach, T., Pease, S., Fraser, S. E. and Pantazis, P. (2011). Oct4 kinetics predict cell lineage patterning in the early mammalian embryo. Nat. Cell Biol. 13, 117-123.

Quinones-Coello, A. T., Petrella, L. N., Ayers, K., Melillo, A., Mazzalupo, S., Hudson, A. M., Wang, S., Castiblanco, C., Buszczak, M., Hoskins, R. A. et al. (2007) Exploring strategies for protein trapping in Drosophila. Genetics 175, 1089-1104.

Rao, C. V. and Arkin, A. P. (2003). Stochastic chemical kinetics and the quasisteady-state assumption: application to the Gillespie algorithm. J. Chem. Phys. 118, 4999-5010.

Reed, H. C., Hoare, T., Thomsen, S., Weaver, T. A., White, R. A., Akam, M. and Alonso, C. R. (2010). Alternative splicing modulates Ubx protein function in Drosophila melanogaster. Genetics 184, 745-758.

Saadaoui, M., Merabet, S., Litim-Mecheri, I., Arbeille, E., Sambrani, N., Damen W., Brena, C., Pradel, J. and Graba, Y. (2011). Selection of distinct HoxExtradenticle interaction modes fine-tunes Hox protein activity. Proc. Natl. Acad. Sci. USA 108, 2276-2281.

Sanchez, A., Garcia, H. G., Jones, D., Phillips, R. and Kondev, J. (2011). Effect of promoter architecture on the cell-to-cell variability in gene expression. PLoS Comput. Biol. 7, e1001100.

Sarov, M., Barz, C., Jambor, H., Hein, M. Y., Schmied, C., Suchold, D., Stender, B., Janosch, S., KJ, V. V., Krishnan, R. T. et al. (2016). A genome-wide resource for the analysis of protein localisation in Drosophila. eLife 5, e12068.

Schindelin, J., Arganda-Carreras, I., Frise, E., Kaynig, V., Longair, M., Pietzsch, T., Preibisch, S., Rueden, C., Saalfeld, S., Schmid, B. et al. (2012). Fiji: an open-source platform for biological-image analysis. Nat. Methods 9, 676-682. 
Schwanhäusser, B., Busse, D., Li, N., Dittmar, G., Schuchhardt, J., Wolf, J., Chen, W. and Selbach, M. (2011). Global quantification of mammalian gene expression control. Nature 473, 337-342.

Sela, I. and Lukatsky, D. B. (2011). DNA sequence correlations shape nonspecific transcription factor-DNA binding affinity. Biophys. J. 101, 160-166.

Shimoga, V., White, J. T., Li, Y., Sontag, E. and Bleris, L. (2013). Synthetic mammalian transgene negative autoregulation. Mol. Syst. Biol. 9, 670.

Slattery, M., Voutev, R., Ma, L., Nègre, N., White, K. P. and Mann, R. S. (2013). Divergent transcriptional regulatory logic at the intersection of tissue growth and developmental patterning. PLoS Genet. 9, e1003753.

Smolander, O.-P., Kandhavelu, M., Mannerström, H., Lihavainen, E., Kalaichelvan, S., Healy, S., Yli-Harja, O., Karp, M. and Ribeiro, A. S. (2011) Cell-to-cell diversity in protein levels of a gene driven by a tetracycline inducible promoter. BMC Mol. Biol. 12, 21.

Soltani, M., Bokes, P., Fox, Z. and Singh, A. (2015). Nonspecific transcription factor binding can reduce noise in the expression of downstream proteins. Phys. Biol. 12, 055002

Stroeher, V. L., Gaiser, J. C. and Garber, R. L. (1988). Alternative RNA splicing that is spatially regulated: generation of transcripts from the Antennapedia gene of Drosophila melanogaster with different protein-coding regions. Mol. Cell. Biol. 8 , 4143-4154.

Swain, P. S., Elowitz, M. B. and Siggia, E. D. (2002). Intrinsic and extrinsic contributions to stochasticity in gene expression. Proc. Natl. Acad. Sci. USA 99, 12795-12800.

Szaloki, N., Krieger, J. W., Komaromi, I., Toth, K. and Vamosi, G. (2015). Evidence for homodimerization of the c-Fos transcription factor in live cells revealed by fluorescence microscopy and computer modeling. Mol. Cell. Biol. 35 , 3785-3798.
Taniguchi, Y., Choi, P. J., Li, G. W., Chen, H., Babu, M., Hearn, J., Emili, A. and Xie, X. S. (2010). Quantifying E. coli proteome and transcriptome with singlemolecule sensitivity in single cells. Science $\mathbf{3 2 9}, 533-538$.

Thattai, M. and van Oudenaarden, A. (2001). Intrinsic noise in gene regulatory networks. Proc. Natl. Acad. Sci. USA 98, 8614-8619.

Tiwari, M., Mikuni, S., Muto, H. and Kinjo, M. (2013). Determination of dissociation constant of the NFkappaB p50/p65 heterodimer using fluorescence cross-correlation spectroscopy in the living cell. Biochem. Biophys. Res. Commun. 436, 430-435.

Tsutsumi, M., Muto, H., Myoba, S., Kimoto, M., Kitamura, A., Kamiya, M., Kikukawa, T., Takiya, S., Demura, M., Kawano, K. et al. (2016). In vivo fluorescence correlation spectroscopy analyses of FMBP-1, a silkworm transcription factor. FEBS Open Biol. 6, 106-125.

Venken, K. J., Schulze, K. L., Haelterman, N. A., Pan, H., He, Y., Evans-Holm, M., Carlson, J. W., Levis, R. W., Spradling, A. C., Hoskins, R. A. et al. (2011). MiMIC: a highly versatile transposon insertion resource for engineering Drosophila melanogaster genes. Nat. Methods 8, 737-743.

Vukojevic, V., Heidkamp, M., Ming, Y., Johansson, B., Terenius, L. and Rigler, R. (2008). Quantitative single-molecule imaging by confocal laser scanning microscopy. Proc. Natl. Acad. Sci. USA 105, 18176-18181.

Vukojevic, V., Papadopoulos, D. K., Terenius, L., Gehring, W. J. and Rigler, R. (2010). Quantitative study of synthetic Hox transcription factor-DNA interactions in live cells. Proc. Natl. Acad. Sci. USA 107, 4093-4098.

Zechner, C., Ruess, J., Krenn, P., Pelet, S., Peter, M., Lygeros, J. and Koeppl, H. (2012). Moment-based inference predicts bimodality in transient gene expression. Proc. Natl. Acad. Sci. USA 109, 8340-8345.

Zink, B., Engström, Y., Gehring, W. J. and Paro, R. (1991). Direct interaction of the Polycomb protein with Antennapedia regulatory sequences in polytene chromosomes of Drosophila melanogaster. EMBO J. 10, 153-162. 